\title{
Semantics-driven extraction of timed automata from Java programs
}

\section{Giovanni Liva1 (D) - Muhammad Taimoor Khan² ${ }^{2}$ Martin Pinzger ${ }^{1}$}

Published online: 22 March 2019

(C) The Author(s) 2019

\begin{abstract}
The automatic verification of time properties of models extracted from programs is challenging, mainly because modern programming languages, such as Java, represent time without a proper semantics. Current approaches to extract time models from source code either represent time only as a tree-like sequence of events or require developers to manually provide a formal model of the time behavior. This makes it difficult for software developers to verify various aspects of their systems, such as timeouts, delays and periodicity of the execution. In this paper, we introduce a formal definition of the time semantics for the Java programming language. Based on the semantics, we present an approach to automatically extract timed automata and their time constraints from Java programs at method level. First, our approach detects the Java statements that involve time, from which it then extracts the timed automata. Our extracted automata are directly amenable to the verification of time properties of the corresponding Java methods. We evaluated the accuracy of our approach on twenty open source Java projects that implement time behavior in their source code. The results show that our approach achieves $100 \%$ precision and recall in identifying time related information. They also show that $95 \%$ of the timed automata extracted from source code correctly model the time behavior of the method. Finally, we show the applicability of our timed automata to identify eight real errors in four open source Apache systems.
\end{abstract}

Keywords Program verification · Time semantics · Timed automata

Communicated by: Coen De Roover, David Lo and Jianjun Zhao

Giovanni Liva

giovanni.liva@aau.at

Muhammad Taimoor Khan

m.t.khan@surrey.ac.uk

Martin Pinzger

martin.pinzger@aau.at

1 Software Engineering Research Group, Alpen-Adria Universität Klagenfurt, Klagenfurt, Austria

2 Surrey Center for Cyber Security, University of Surrey, Guildford, UK 


\section{Introduction}

The quality of software is mainly determined by automatically testing or manually reviewing the software. These activities consume between $40 \%$ and $67 \%$ of the total project cost depending on the maturity level of the company (Laporte et al. 2012). Moreover, while these activities help developers to detect the presence of errors they fail to assure the absence of errors in software as said by Dijkstra (1972). A more rigorous way to establish software quality is to formally assure that the software is free of critical errors using formal methods. Formal methods require an abstract model of the source code that captures the specific semantics of the implemented functionalities. A particular domain of such a functionality is the time domain. For instance, developers use time to implement time frames when external events are expected to occur, to set execution timeouts, or to schedule events that occur periodically. A common technique to specify and model the time behavior of programs is the timed automata formalism.

The formal verification of time properties of programs, modeled as timed automata, has been intensively studied over the last 20 years. Timed automata have been introduced by Alur (1999) and allow software engineers to model and analyze the timing behavior of programs.

For instance, Jayaraman et al. (2015) used the timed automata modeled by the developers to monitor the subject system and to verify whether its execution conforms to its specification. Hakimipour et al. (2010) present an approach to derive real-time Java programs from the timed automata constructed by the users. Following the same idea, Georgiou et al. (2013) presents a technique to translate timed input/output automata into executable Java code. All these research efforts propose techniques that require the developers to provide the formal models and, typically, they are constructed manually.

Some recent approaches, such as presented in Lo et al. (2009) and Walkinshaw and Bogdanov (2008), automatically extract such models from Java source code. However, these approaches do not consider the specific semantics of the programming language and fail to correctly model the time domain in programs. For instance, they only retain time as a sequence of events represented in a tree-like structure but fail to model other time aspects of the program, such as timing delays.

In this paper, we address two shortcomings of the aforementioned approaches: (i) they are based on an informal semantics of time and (ii) the timed automata are manually constructed and thus, they are error prone. We propose a technique to automatically extract timed automata from source code by introducing a formal time semantics for the Java programming language. The extracted timed automata are directly amenable to verification aiding developers to verify the correctness of their implementation.

Figure 1 shows an overview of our approach to extract the timed automata. Based on our defined semantics of time, first we parse the source code of a Java method and extract its time related information. Next, we use the extracted time information to build the states and transitions that defines the timed automaton.

Our approach works at method level therefore we need a way to model side effects caused by using non-local time variables, such as class attributes. Our approach tackles this problem with dynamic analysis monitoring the values of these statements and expressions during the execution of the test suite. For each run of a test case, our approach generates a set of instances of the timed automaton that can be used for verification. 


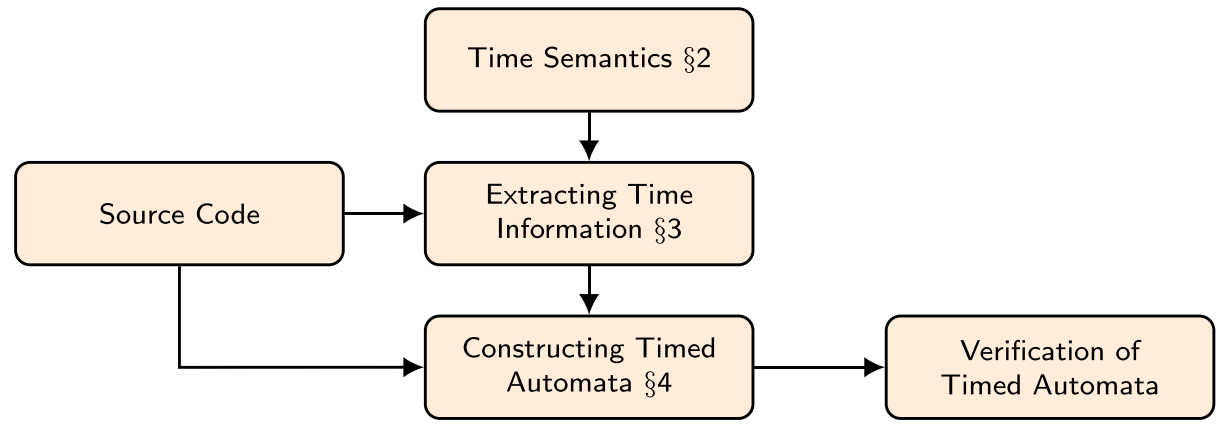

Fig. 1 Overview of our approach to extract a timed automaton of a Java method

We have implemented our approach in a prototype tool ${ }^{1}$ used to show and study its applicability. We evaluated our approach on 20 open source Java projects. Our experiments demonstrate that the produced timed automata have a precision and recall of $100 \%$ in covering time related information. Furthermore, $95 \%$ of the timed automata extracted from source code correctly model the time behavior of a method. In addition, the results show that our semantics of time for the Java APIs is sufficient since, in our 20 subject systems, we found that the developers only use the methods provided by the Java APIs to implement time-related behavior. The maximum time for generating a timed automaton took less than 0.5 seconds and had a low memory footprint. Finally, the results show that our timed automata could effectively be used to identify 8 real errors in four open source Java projects.

In summary, this paper makes the following contributions:

- a formal definition of the semantics for the Java 8 (and later) time APIs;

- an approach to automatically extract timed automata for Java methods;

- an evaluation of our approach with 20 open source Java projects;

- $\quad$ an open source implementation of our approach in a prototype tool.

This paper extends our SCAM 2017 paper "Extracting Timed Automata from Java Methods" (Liva et al. 2017). In this new version, we have improved the time semantics, addressing several limitations of the original approach and we have extended the previous experiments from 10 to 20 open source Java projects. Moreover, we have added three more research questions (RQ2, RQ3, and RQ5) to further analyze the limitations of our approach. Finally, we have added a description of the methodology to proof the soundness of our translation.

The remainder of the paper is organized as follows: Section 2 presents our time semantics defined for the Java 8 methods. Section 3 presents the application of our semantics to extract time information from Java statements. Section 4 introduces our approach to extract the timed automata from Java methods. Section 5 presents the evaluation. The discussion of the results and threats to validity are presented in Section 6. Section 7 presents related work and we conclude the paper in Section 8 .

\footnotetext{
${ }^{1}$ https://git-isys.aau.at/giliva/java-time-verification
} 


\section{Time Semantics}

In this section, we first provide background information on the semantics of programming languages. Next, we present our manual categorization of the Java 8 (and later) time APIs. Based on the categorization, we then introduce our time semantics for the Java language.

\subsection{Programming Languages and Semantics}

The idea of using semantics as a tool for modelling program behaviour is rooted in the work of McCarthy (1993) that was used to model the ALGOL (McCarthy 1964) and LISP (Steel 1969) programming languages. Based on this work, Plotkin $(1977,1981)$ developed a semantics that capture the notion of a computational step. This approach has been successfully used for formalizing semantics for various programming languages and computational models (Abadi and Cardelli 2012; Cardelli and Gordon 1998; Flatt et al. 1998; Hennessy 1988; Milner 1999; Sangiorgi and Walker 2003; Wright and Felleisen 1994). Nowadays, all the mainstream languages have their formal semantics, e.g., PHP (Filaretti and Maffeis 2014), C (Hathhorn et al. 2015), C\# (Börger et al. 2005), Python (Guth 2013), and Javascript (Park et al. 2015). The definition of a formal semantics permits to address different problems, such as the detection of inconsistencies in programs (Liva et al. 2018), the detection of inconsistencies in the implementation of compilers (Tristan 2009), and monitoring the reliability and security of industrial controllers (Khan et al. 2018).

Regarding the Java programming language, three different semantics were proposed: ASM-Java (Stärk et al. 2012), JavaFAN (Farzan et al. 2004), and K-Java (Bogdanas and Roşu 2015). ASM-Java was the first complete semantics of Java. It defines a complete semantics for both, the Java language and its bytecode interpretation for the version 1.0 of the programming language. JavaFAN defines only the semantics of the most frequently used features of Java 1.4.

The most recent and richest semantics for Java is provided by K-Java presented by Bogdanas and Roşu (2015). They proposed a complete definition of semantics for the Java 1.4 language. In this work, we enrich their definition including the semantics of time for the Java programming language.

All the semantics presented above define the meaning of the structure of a program but they do not provide a meaning for the domain on which the program statements operate.

With respect to the work presented in this paper, this domain is time. We present a time semantics for the Java programming language that is a rigorous mathematical study of the meaning of the time APIs offered by the language. The semantics describes the processes that the Java Virtual Machine follows when executing the functionality of such APIs. Like many other programming languages, Java provides developers with APIs to implement time related behaviour, such as the Java java.time. LocalDate class. But, Java also provides APIs that allow developers to represent and handle time and timestamps with integer values.

The integer representation of time can introduce semantic inconsistencies in a program resulting in failures or preemptive termination. For example, the Java statement long now $=-1$, is syntactically correct and it compiles. However, during the execution of the program, if the variable now is used in a timed API call, it will result in a runtime exception. The error is not detected by the Java compiler because it does not understand the semantics of time and that the variable now is a time variable since it is used in a timed API call. Therefore, the compiler fails to warn the developer that the variable now holds an incorrect time value, namely -1 . 
As a prerequisite for defining a semantics of time for the Java programming language, we first need to find out which basic methods of Java deal with time, meaning return time or consume time as a parameter. This is presented in the next subsection.

\subsection{Classification of Time Related Java 8 Methods}

For defining the time semantics, we manually analyzed the API documentation of Java 8 classes and identified all API methods that either return time as an integer value or consume time as an integer value in one of their parameters. We also performed this analysis with the API documentation of the Java versions 9 and 10 and we discovered that the time APIs did not change. Therefore, our semantics support also the most recent versions of Java. Next, we classified the found methods into the following four categories:

1. Return Time (RT): The first category covers methods that return an integer value that represents time. For example, the static currentTimemillis () method of the System class returns the current time in milliseconds.

2. Explicit Time (ET): The second category covers methods that contain a time parameter declared as integer. For example, in the connect method of the Socket class, if called with two parameters, the second parameter specifies the maximum amount of time for establishing the connection.

3. Explicit Wait (EW): The third category is composed of methods that wait for an external event to continue the execution of a thread. Since it is possible that this event might never occur, these methods can potentially block the execution of a thread forever. Examples are the wait () method of the Object class or the method get of the Future class.

4. Set Timeout (ST): The fourth category comprises methods which change their behavior with a time constraint that is set by a preceding method call. For example, the connection method of the URLConnection class by default has no upper time limit for establishing the connection. Instead, a timeout can be set by calling the setConnect Timeout method before calling the connection method.

Table 1 presents an excerpt ${ }^{2}$ of the analyzed Java 8 APIs methods that involve time. In this paper, we focus on the first three categories of time related methods and we provide a semantics for them. For the category $\mathbf{S T}$, it is not always possible, using static analysis, to correctly detect whether a method call has a specific timeout set by a preceding method call. Therefore, we skip this category of methods for this work and will address them in our future work.

\subsection{Semantics of Java Time Statements}

Based on the classification of time related methods of the Java 8 APIs, we introduce the semantics of time for the statements in Java programs. We start with introducing the general concepts for representing time variables and time related methods.

A time variable is a program variable, which stores time values. We model the time as positive natural number and we define the set of time variables as $V^{t}$, such that $\forall v \in$ $V^{t}$. value $(v) \in \mathbb{N}^{+}$, where value $(\cdot)$ refers to the value held by the variable $v$. We define a set of Java method definitions with the letter $M$ where the superscript $t$ is used to define

\footnotetext{
${ }^{2}$ The full list is available at https://git.io/fAbZR
} 
Table 1 Excerpt of time related methods found in the Java 8 APIs

\begin{tabular}{|c|c|c|c|}
\hline Class & Method signature & Class & Method signature \\
\hline Return Time (RT) & & Explicit Time (ET) & \\
\hline System & nanoTime() & Socket & connect(SocketAddress,int) \\
\hline System & currentTimeMillis() & Thread & join(long) \\
\hline Clock & millis() & Thread & join(long,int) \\
\hline Duration & get(TemporalUnit) & Thread & sleep(long) \\
\hline Duration & getNano() & Thread & sleep(long,int) \\
\hline Duration & getSeconds() & FutureTask & get(long,TimeUnit) \\
\hline$\cdots$ & $\cdots$ & $\cdots$ & $\cdots$ \\
\hline Explicit Wait (EW) & & Set Timeout (ST) & \\
\hline Object & wait() & InputStream & $\operatorname{read}()$ \\
\hline Future & $\operatorname{get}()$ & InputStream & read(byte[]) \\
\hline FutureTask & $\operatorname{get}()$ & InputStream & read(byte[],int,int) \\
\hline Thread & join () & HttpURLConnection & connect() \\
\hline Process & waitFor() & ServerSocket & $\operatorname{accept}()$ \\
\hline CountDownLatch & await() & SSLServerSocket & $\operatorname{accept}()$ \\
\hline$\cdots$ & $\cdots$ & $\cdots$ & $\cdots$ \\
\hline
\end{tabular}

that they are time related and the subscript is used to define the category to which a method belongs: $M_{r t}^{t}$ denotes the set of RT methods that return time; $M_{e t}^{t}$ denotes the set of ET methods that have a time parameter in their signature; and $M_{e w}^{t}$ denotes the set of EW methods that can potentially block the execution of a thread forever. Finally, we call the quadruplet $V^{t}, M_{r t}^{t}, M_{e t}^{t}$, and $M_{e w}^{t}$ an environment and we denote it with the letter $E$. The environment keeps track of the time information extracted from the source code.

$$
E=\left\langle V^{t}, M_{r t}^{t}, M_{e t}^{t}, M_{e w}^{t}\right\rangle
$$

We define the time semantics as a set of operational semantics (Plotkin 1981) rules. A rule has some premises that constitute the preconditions to apply the rule. The premises have an environment $E$ and the program statement $S$. Based on this, we can apply the rules $R_{1}, \ldots, R_{n}$ that can conclude a new environment $E^{\prime}$.

$$
\frac{R_{1}(E, S), \ldots, R_{n}(E, S) \vdash\left\langle E^{\prime}\right\rangle}{\langle E, S\rangle \vdash\left\langle E^{\prime}\right\rangle}
$$

Given the time domain $\mathbb{T}$ defined as positive integer numbers and the current point in time $t_{0} \in \mathbb{T}$, we define the rules $T_{1}, T_{2}$, and $T_{3}$ to model the time semantics of the three categories of methods considered by our approach. These three rules describe how the time changes given the couple $\left(V^{t}, t_{0}\right)$, that represent the list of time variables and the current point in time, and the API method call. We define $\operatorname{val}(\cdot)$ as the function which returns the value of the input expression that can be either a reference to a variable or a method call. The rule $T_{1}$ handles the assignment of time returned by calls to methods of the category $\mathbf{R T}$ 
updating the value stored by the time variable $x$ without touching the execution time. It is defined as:

$$
T_{1}: \frac{x \in V^{t} \quad m \in M_{r t}^{t}}{\left\langle\left(V^{t}, t_{0}\right), x=m\left(\_\right)\right\rangle \vdash\left\langle V^{t}\left[x \backslash \operatorname{val}\left(m\left(\_\right)\right)\right], t_{0}\right\rangle}
$$

Rule $T_{2}$ handles ET method calls containing a time argument represented by the variable $t$. At the end of the execution of the method $m$, the time is increased by value $t$ :

$$
T_{2}: \frac{m \in M_{e t}^{t}}{\left\langle\left(V^{t}, t_{0}\right), x=m\left(, t, \_\right)\right\rangle \vdash\left\langle V^{t}, t_{0}+\operatorname{val}(t)\right\rangle}
$$

Finally, rule $T_{3}$ handles calls to $\mathbf{E W}$ methods that could potentially block the execution of a thread forever. It is defined as:

$$
T_{3}: \frac{m \in M_{e w}^{t}}{\left\langle\left(V^{t}, t_{0}\right), x=m\left(, t, \_\right)\right\rangle \vdash\left\langle V^{t}, \infty\right\rangle}
$$

In the next section, we present how our time semantics is used to infer time related Java methods, statements, and time variables.

\section{Extracting Time Information}

Based on the time semantics presented in the previous section, we define a set of rules to analyze the source code of a project. Through the analysis, our approach gathers the methods and statements that are time related and program variables that are time variables. We group the rules into three categories:

- time methods analysis,

- branching statements analysis,

- expressions analysis.

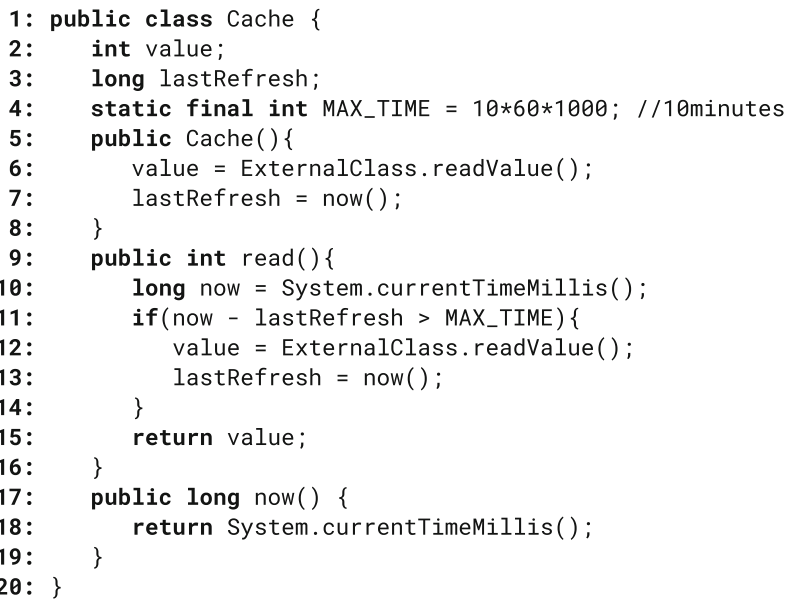

Fig. 2 Code example that uses the Java time API 
Note, these three categories of rules are applied to the source code until no more time methods, statements, or time variables are found. Vice versa, when new time methods, statements, or variables are found, then the additional information is used to perform another analysis round.

Figure 2 shows an example of using the time API in Java. The class Cache implements a simple cache that stores the value returned by the invocation of Externalclass.readValue (). When the method read () is called, the code verifies how much time elapsed since the last call to readValue (). If the elapsed time is greater than a threshold MAX_TIME, the cached value is refreshed. We use this example to show how our semantics is applied to determine time methods, statements, and variables.

\subsection{Time Methods Analysis}

Using the definitions given in Section 2.3, we first introduce a set of rules to gather the methods of the categories RT and ET in the source code of a Java program. Methods that return time are added to $M_{r t}^{t}$. Methods that contain a time variable as a parameter are added to $M_{e t}^{t}$. Note, since we did not provide a formal semantics for $\mathbf{S T}$ methods, our approach does not consider this category of methods. Furthermore, while our approach supports the Java APIs EW methods, it does currently not cover EW methods implemented by developers.

For gathering the developer's defined RT and ET methods, our approach applies the rules $R_{r t}$ and $R_{e t}$ detailed in the following.

Rule $\boldsymbol{R}_{\boldsymbol{r t}}$ This rule matches the project's RT methods that return time and adds them to the set $M_{r t}^{t}$. It is defined as:

$$
\begin{gathered}
\left\langle\left(V^{t}, M_{r t}^{t}, M_{e t}^{t}, M_{e w}^{t}\right), S\right\rangle \vdash\left\langle V^{t^{\prime}}, M_{r t}^{t}, M_{e t}^{t}, M_{e w}^{t}\right\rangle \\
R_{r t}: \frac{\exists r: \operatorname{return}(S) .\left(i s \operatorname{Var}(r) \wedge r \in V^{t^{\prime}}\right) \vee\left(i s \operatorname{Call}(r) \wedge r \in M_{r t}^{t}\right)}{\left\langle\left(V^{t}, M_{r t}^{t}, M_{e t}^{t}, M_{e w}^{t}\right), \operatorname{name}(\text { pars }) S\right\rangle \vdash\left\langle V^{t},\{\text { name }\} \cup M_{r t}^{t}, M_{e t}^{t}, M_{e w}^{t}\right\rangle}
\end{gathered}
$$

Given a method called name with the list of parameters pars and the method body $S$, our approach applies the time semantics recursively to the body $S$. If $S$ contains a return statement that references a time variable or a call to an RT method, the method name is added to the set $M_{r t}^{t}$. The function $i s \operatorname{Var}(\cdot)$ returns true if the expression is a reference to a variable. Similarly, is Call(.) returns true if the expression is a method call. The function return (.) obtains the return statements of the given method body $S$.

Example Considering the method now defined at Line 17 of Fig. 2, the rule $R_{r t}$ is matched as follows:

$$
\begin{aligned}
& \left\langle\left(,, M_{r t}^{t}, \ldots, \ldots\right),\{\text { return sys.currTime }() ;\}\right\rangle \vdash\left\langle \_, M_{r t}^{t}, \ldots,\right\rangle \\
& R_{r t}: \frac{r:=\operatorname{Sys.currTime}() \cdot\left(\_\vee\left(i \operatorname{Call}(r) \wedge r \in M_{r t}^{t}\right)\right)}{\left\langle\left(\ldots, M_{r t}^{t}, \ldots, \_\right), \text {now }()\{\text { return Sys.currTime }() ;\}\right\rangle \vdash\left\langle \_,\{\text {now }\} \cup M_{r t}^{t}, \ldots, \_\right\rangle}
\end{aligned}
$$

The premise of the rule $R_{r t}$ matches the variable name with the method name now, the list of parameter pars with an empty list, and the body $S$ with the return statement of the method. For readability, we have shortened the method call to System. currentTimeMillis () in the return statement. The rule analyzes the body of the method marking the method call in the return statement as an RT method call. When the rule retrieves the expression of the return statement in the variable $r$, it validates the second branch of the $o r$ 
clause satisfying all the judgments of the rule. Therefore, the fully qualified name of method now is added to the list of RT methods.

Rule $\boldsymbol{R}_{\boldsymbol{e}}$ This rule identifies the project's ET methods that accept a time parameter and adds them to the set $M_{e t}^{t}$.

It is defined as:

$$
R_{e t}: \frac{\left\langle\left(V^{t}, M_{r t}^{t}, M_{e t}^{t}, M_{e w}^{t}\right), S\right\rangle \vdash\left\langle V^{t \prime}, M_{r t}^{t}, M_{e t}^{t}, M_{e w}^{t}\right\rangle \quad \exists p: \text { pars.p } \in V^{t^{\prime}}}{\left\langle\left(V^{t}, M_{r t}^{t}, M_{e t}^{t}, M_{e w}^{t}\right), \operatorname{name}(\text { pars })\{S\}\right\rangle \vdash\left\langle V^{t^{\prime}}, M_{r t}^{t},\{\text { name }\} \cup M_{e t}^{t}, M_{e w}^{t}\right\rangle}
$$

Given a method called name with the list of parameters pars and the method body $S$, our approach adds it to $M_{e t}^{t}$ if the set of time variables $V^{t \prime}$ of the environment, resulting from the application of our time semantics to the body $S$, contains a parameter declared by method name.

Both rules, $R_{r t}$ and $R_{e t}$, require the application of our time semantics to the statements $S$ of the method body to determine whether a method is of category RT or ET. Our approach applies the time semantics to the statements following the order of the method's control flow.

Java provides different types of statements, such as conditional and loop statements, lambda expressions, ternary operator, assignment and mathematical expressions. In the next subsections, we provide the rules to handle the relevant Java statements. The other types of statements are handled by our approach in a similar way therefore we omit the rules for them.

\subsection{Branching Statements Analysis}

In this subsection, we present the relevant rules for the two branching statements if and while. The other branching statements of Java, such as try-catch and do-while, follow the same approach.

Rules $\boldsymbol{R}_{\text {if }}$ and $\boldsymbol{R}_{\text {loop }}$ The idea of the rules $R_{\text {if }}$ and $R_{\text {loop }}$ is to first apply the time semantics to the guard $B$ and then to the body $S$, collecting the time information. Our approach applies these rules until a fix point is reached and no more time information is collected in the environment. For the if branching statement, the time information collected from both branches is unified.

$$
\begin{gathered}
R_{\text {if }}: \frac{\langle E, B\rangle \vdash\left\langle E_{0}\right\rangle \quad\left\langle E_{0}, S^{\prime}\right\rangle \vdash\left\langle E^{\prime}\right\rangle \quad\left\langle E_{0}, S^{\prime \prime}\right\rangle \vdash\left\langle E^{\prime \prime}\right\rangle}{\left\langle E,\left(\text { if }(B)\left\{S^{\prime}\right\} \text { else }\left\{S^{\prime \prime}\right\}\right)\right\rangle \vdash\left\langle E^{\prime} \cup E^{\prime \prime}\right\rangle} \\
R_{\text {loop }}: \frac{\langle E, B\rangle \vdash\left\langle E^{\prime}\right\rangle \quad\left\langle E^{\prime}, S\right\rangle \vdash\left\langle E^{\prime \prime}\right\rangle}{\langle E,(\text { while }(B)\{S\})\rangle \vdash\left\langle E^{\prime \prime}\right\rangle}
\end{gathered}
$$

Example Considering the if statement in Lines 11-14 of Fig. 2, the rule $R_{i f}$ matches as follows:

$$
R_{i f}: \frac{\left\langle E_{0}, S\right\rangle \vdash\left\langle E^{\prime}\right\rangle \quad\left\langle E_{0}, \emptyset\right\rangle \vdash\left\langle E_{0}\right\rangle}{\langle E,(\boldsymbol{i} \text { (now-lastRefresh }>\text { MAX_TIME) }\{S\} \text { else }\{\})\rangle \vdash\left\langle E^{\prime} \cup E_{0}\right\rangle}
$$

The guard $B$ matches the expression now - lastRefresh > MAX_TIME, the thenbody $S^{\prime}$ matches the list of statements in Lines 12-13, and the else-body $S^{\prime \prime}$ matches an empty list of statements. For readability, we omit the statements in the then-body. 
The rule first analyzes the guard expression recursively using the rules presented in the next subsection. The result is an environment $E^{\prime}$ with the time variables referenced in the guard expression. Then, the rule uses the environment $E$ as input to analyze the then- and else-body recursively. Since the else-body is empty, the resulting environment does not change.

\subsection{Expressions Analysis}

The following presents the set of rules to analyze Java expressions to identify time related variables. First, we present how to process boolean expressions and then how to process assignment and mathematical expressions.

In a boolean expression, there are two different cases in which our approach marks a program variable as time variable: (i) the variable is compared with a time variable or (ii) the variable is compared with the result of an RT method call. If an expression consists of multiple boolean expressions, we process each expression separately. Considering the if statement at Line 11 of Fig. 2, given that variable now defined in Line 10 is a time variable, our semantics analyzes first the boolean guard and then the then-branch. In the guard, the variable now is compared with the constant MAX_TIME and thus, our semantics marks the constant MAX_TIME as time related variable.

Regarding assignment and mathematical expressions, there are three different cases in which a programming variable is used as time variable:

- The variable is assigned the result of an RT method. This case is handled by the rule $R_{1}$.

- The variable is used as timeout parameter in an ET method call. This case is handled by the rule $R_{2}$.

- The variable is used in a mathematical expression with other time variables or time methods. This case is handled by the rule $R_{3}$.

Rule $R_{1}$ The rule $R_{1}$ verifies that the return value of method $m$ is assigned to the variable $x$ and $m$ is a method of the RT category. If this condition holds, $x$ is added to the set of time variables $V^{t}$.

$$
R_{1}: \frac{m \in M_{r t}^{t}}{\left\langle\left(V^{t}, M_{r t}^{t}, M_{e t}^{t}, M_{e w}^{t}\right), x=m(-)\right\rangle \vdash\left\langle\{x\} \cup V^{t}, M_{r t}^{t}, M_{e t}^{t}, M_{e w}^{t}\right\rangle}
$$

Example Line 10 in Fig. 2 shows a statement on which this rule applies. Variable now is assigned the result of the call to the RT method System. currentTimeMillis () and therefore, our semantics adds now as time variable.

$$
R_{1}: \frac{\text { Sys.currTime }() \in M_{r t}^{t}}{\left\langle\left(V^{t}, M_{r t}^{t}, M_{e t}^{t}, M_{e w}^{t}\right), \mathrm{x}=\text { Sys.currTime }()_{i}\right\rangle \vdash\left\langle\{x\} \cup V^{t}, M_{r t}^{t}, M_{e t}^{t}, M_{e w}^{t}\right\rangle}
$$

Rule $\boldsymbol{R}_{\mathbf{2}}$ For the second case, we define two auxiliary functions to simplify the readability of our semantics. Function $\operatorname{pos}(\cdot)$ returns the position of the input parameter in the method call and function timeoutpars $(\cdot)$ returns the set of indices of the time parameters of the input 
method. The rule marks as time variable every variable that is passed as a time parameter in a method call of the category $\mathbf{E T}$.

$$
R_{2}: \frac{m \in M_{e t}^{t} \wedge \operatorname{pos}(y) \in \operatorname{timeoutpars}(m)}{\left\langle\left(V^{t}, M_{r t}^{t}, M_{e t}^{t}, M_{e w}^{t}\right), m\left({ }_{-}, y,-\right)\right\rangle \vdash\left\langle\{y\} \cup V^{t}, M_{r t}^{t}, M_{e t}^{t}, M_{e w}^{t}\right\rangle}
$$

Rule $\boldsymbol{R}_{\mathbf{3}}$ The last case requires to consider different types of operands of mathematical expressions:

1. Both operands are time variables.

2. One operand is a time variable and the other is a normal variable.

3. One operand is a time variable and the other is a scalar value.

4. One operand is a normal variable and the other is an RT method call.

5. Both operands are RT method calls.

6. One operand is an RT method call and the other is a scalar value.

Each case is encoded in one of the following rules, where $o p_{i}$ is an operand of the mathematical expression:

$$
\begin{aligned}
& e_{1}=o p_{1} \in V^{t} \wedge o p_{2} \in V^{t} \\
& e_{2}=\left(o p_{1} \in V^{t} \vee o p_{2} \in V^{t}\right) \wedge\left(i s \operatorname{Var}\left(o p_{1}\right) \vee i s \operatorname{Var}\left(o p_{2}\right)\right) \\
& e_{3}=\left(o p_{1} \in V^{t} \wedge o p_{2} \in \mathbb{N}^{+}\right) \vee\left(o p_{1} \in \mathbb{N}^{+} \wedge o p_{2} \in V^{t}\right) \\
& e_{4}=\left(i s \operatorname{Var}\left(o p_{1}\right) \wedge o p_{2} \in M_{r t}^{t}\right) \vee\left(o p_{1} \in M_{r t}^{t} \wedge i s \operatorname{Var}\left(o p_{2}\right)\right) \\
& e_{5}=o p_{1} \in M_{r t}^{t} \wedge o p_{2} \in M_{r t}^{t} \\
& e_{6}=\left(o p_{1} \in M_{r t}^{t} \wedge o p_{2} \in \mathbb{N}^{+}\right) \vee\left(o p_{1} \in \mathbb{N}^{+} \wedge o p_{2} \in M_{r t}^{t}\right)
\end{aligned}
$$

If any of the previous rules holds, then the variable used in the assignment statement is added to the set of time variables. Moreover, the rule $R_{3}$ checks that the return type of the expression is numeric because Java allows using the "+" operator with other data types, e.g., it can be used to concatenate strings. This control is necessary to avoid adding variables that do not hold time values.

$$
R_{3}: \frac{e_{1} \vee e_{2} \vee e_{3} \vee e_{4} \vee e_{5} \vee e_{6} \quad \odot \in\{+,-, /, *\} \quad \text { is_numeric }\left(o p_{1} \odot o p_{2}\right)}{\left\langle\left(V^{t}, M_{r t}^{t}, M_{e t}^{t}, M_{e w}^{t}\right), x=o p_{1} \odot o p_{2}\right\rangle \vdash\left\langle\left\{x, o p_{1}, o p_{2}\right\} \cup V^{t}, M_{r t}^{t}, M_{e t}^{t}, M_{e w}^{t}\right\rangle}
$$

Example The guard expression in Line 11 of Fig. 2 shows an instance where this rule applies. The left-hand-side of the condition contains the mathematical expression now lastRefresh. Using rule $R_{1}$ in Line 10 , the variable now is added to the set of time variables $V^{t}$. When the guard expression is processed with the rule $R_{3}$, expression $e_{2}$ is satisfied and the return value is numeric. Therefore, the rule adds the variable lastRefresh to the set of time variables.

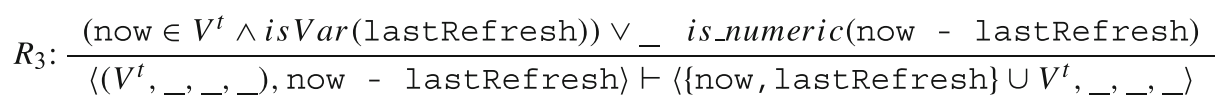




\section{Constructing Timed Automata}

With the time semantics presented in the previous section, our approach is able to extract the time information necessary to generate a timed automaton for a Java method. There are multiple temporal logics available that can be used to describe properties to be verified on the extracted timed automaton. We have decided to use Timed Computation Tree Logic (TCTL) (Baier et al. 2008; Behrmann et al. 2004) because it provides means to describe properties related to time and the execution state and path. Currently, there exist two model checkers for timed automata that support TCTL: Kronos (Yovine 1997) and UPPAAL (Larsen et al. 1997). We decided to use UPPAAL because it has more then 15 years of development and runs on Java 8, while the last release of Kronos dates back to 2002 and we were not able to execute it on our machines. Furthermore, UPPAAL has been successfully used in several case studies, for instance to prove the reliability of gearbox controllers (Lindahl et al. 1998), to correctly synthesize control programs for batch production (Hune et al. 2001), and to verify the correctness of audio protocols (Larsen et al. 1995; Bengtsson et al. 1996).

UPPAAL extends the classical timed automaton theory defined by Alur and Dill (1994) with syntactic sugar, e.g., committed location, and additional features, e.g., integer variables and channels. UPPAAL uses a transition system in which a transition can have three properties: (i) a time constraint, (ii) a reset of time variables, and (iii) an update of variables. Time variables in the context of timed automata are called clock variables. A time constraint specifies an extra condition involving clock variables. A transition is enabled only if the time constraint is satisfied.

The reset option specifies the list of clock variables to reset their values to zero. The update option assigns values to variables. The reset and update actions are performed only when the transition is fired. Moreover, a timed automaton has a set of clock variables that model the flow of time. In our approach, we use a single clock variable $t_{0}$ that keeps track of the execution time.

Our approach to extract UPPAAL timed automata consists of the following three steps:

1. construct the initial timed automaton with states and transitions

2. refine the automaton with additional time constraints; and

3. finalize the automaton.

In the following subsections, we describe each step of our approach in detail.

\subsection{Construct the Initial Timed Automaton}

The construction of the initial timed automaton starts with generating states and transitions following a standard procedure based on the control-flow graph (CFG) of the method's source code.

For each statement node in the CFG, our approach creates a respective committed state in the timed automaton. A committed state freezes the time simulating that the execution of the code is instantaneous. This prevents the interleaving of actions that require atomicity. However, if the CFG node contains a statement with an ET/EW method call, the state created in the timed automaton is a normal state. This models the fact that in this state the time is important and it can elapse. Using committed states helps the model checker to perform internal optimizations and it reduces significantly the state space that it has to explore. Furthermore, we mark the root node of the control-flow graph as initial state of the automaton. 
Similarly, for each connection between two nodes in the CFG, our approach creates a transition that connects the respective states in the automaton. All the transitions extracted in this step are $\epsilon$-transitions without any constraints. The decision of using $\epsilon$-transitions can introduce a problem with branching instructions and resulting in a non-deterministic automaton. Since we are interested only in time properties of the code, which path is taken in an automaton run is not important. However, as presented in detail later, if a branching instruction in the code is guarded by a time expression, our approach adds the time expression as constraint to the transition of the then-branch. Moreover, for each transition from state $s$ to $s^{\prime}$, our approach verifies if state $s$ contains an expression that assigns a value to a time variable as defined by rules $R_{1}$ and $R_{3}$. If this is the case, the assignment is added to the transition as update property.

\subsection{Refine the Automaton with Time Constraints}

After constructing an initial timed automaton for a Java method, our approach analyzes the time statements in the CFG to infer time constraints that are used to refine the transitions of the initial automaton. Currently, our approach infers the following three types of time constraints:

- Timeout Constraint: covers calls to ET methods that contain an argument defining the maximum waiting time, such as Thread. sleep (2000).

- Indefinite Wait Constraint: covers calls to EW methods that can stop the execution of a method forever, such as Thread.join ().

- Time Expired Constraint: covers conditional statements that trigger the execution of a specific piece of code, such as if (ticks $<$ MAX_TICKS) $\{\ldots\}$.

Based on the inferred time constraints, the approach updates the corresponding transition in the initial automaton from state $s$ to state $s^{\prime}$ accordingly.

Timeout Constraint Method calls to ET methods contain an argument that specifies the maximal waiting time for the termination of the method call. This represents a Timeout Constraint that sets the maximum execution time for that method call. For each statement in the CFG that calls a method of the category ET our semantics is used to first extract the timeout parameters. Next, for each timeout parameter timeout our approach adds a time constraint $t_{0}<=$ timeout to all the transitions exiting from state $s$ that represents that method call in the automaton. Moreover, all the transitions that are entering in that state $s$ are modified inserting the reset of variable $t_{0}$. With this model, when the automaton run enters state $s$, the clock variable that keeps track of the execution time will block the execution of the automaton for a maximum of timeout time.

Indefinite Wait Constraint Method calls to EW methods could possibly block the execution of a method forever. If our approach identifies an $\mathbf{E W}$ method call in the CFG, it adds an $\epsilon$-transition from state $s$ to $s$ that represents that method call in the automaton. The self loop models the fact that the automaton can either proceed to the next state or stay forever in the same state.

Time Expired Constraint The condition of a branching instruction can contain a time constraint to decide which branch to execute. An example of such a time constraint is given by the if-condition at Line 11 of Fig. 2. If enough time has passed, it executes the code in the then-branch. Our approach models this using the following algorithm: First, it parses the 
condition and constructs the Abstract Syntax Tree (AST) of the time expression as depicted by the first tree on the left hand-side of Fig. 3. Next, it applies our time semantics to identify expressions that are time related (i.e., contain a time variable or a call to an RT method that returns time). In this example, the expressions e 1 and e 3 are time related. All the nodes that do not contain a time expression are removed from the tree. In our example, the node e 2 is removed. Next, the algorithm removes the nodes representing the boolean operators from which one or both child nodes have been removed in the previous step pushing up the remaining child, if present. In the example shown on the right-hand side of Fig. 3, the node "| |" is removed because its expression e 2 has been removed and e 3 has been pushed up. Finally, the resulting tree is pretty-printed as a string that it is added as a time constraint to the transition representing the then-branch. Furthermore, the negated version of it is added as a time constraint to the transition representing the else-branch. This way our approach creates a deterministic automaton for branching instructions that are time related.

Finally, we translate the refined timed automaton into the corresponding UPPAAL model. However, the translated model may not be amenable to the verification because it may include some expressions which are not directly supported by UPPAAL. We address this issue in the next sub-section.

\subsection{Finalize the Timed Automaton}

The final step of our approach is to rewrite the expressions that have been copied from the source code as correct UPPAAL expressions. Our approach finalizes the previously constructed UPPAAL timed automaton by formalizing those time expressions, which are not supported by UPPAAL, i.e., (i) time variables and (ii) RT method calls. They need to be replaced by concrete values or UPPAAL expressions.

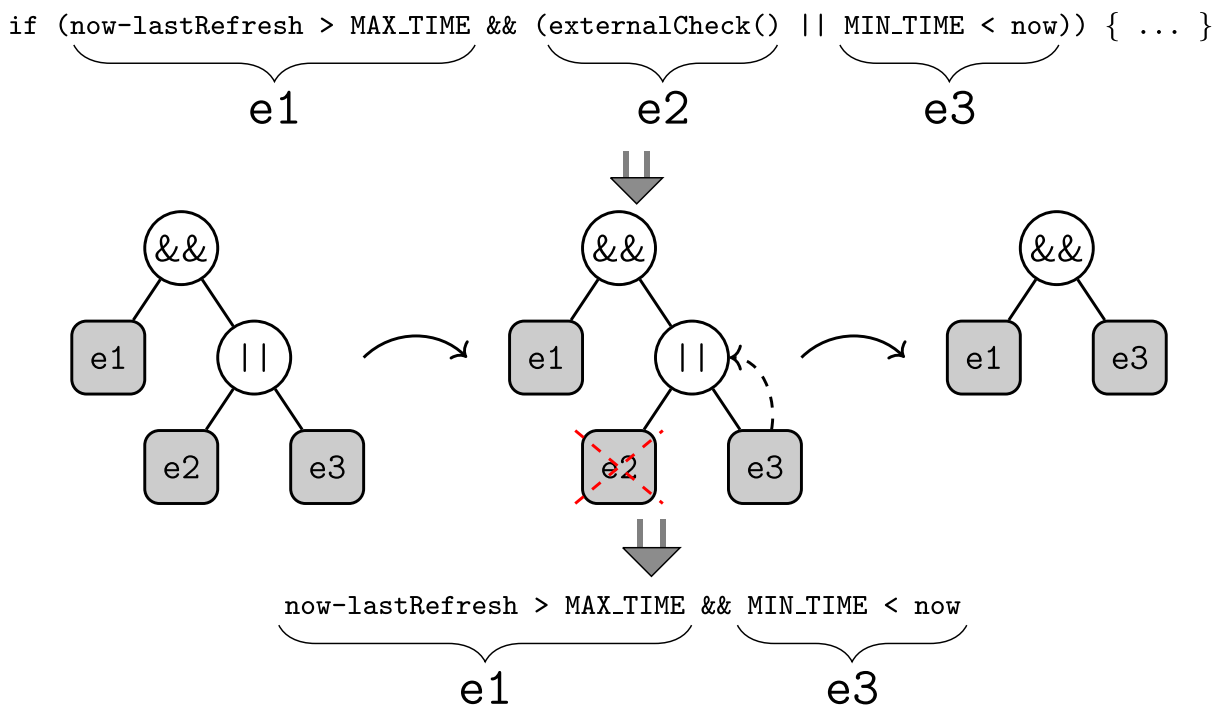

Fig. 3 Example of extracting the time related expression from the if-condition in which e1 and e3 are time related and $e 2$ is not 

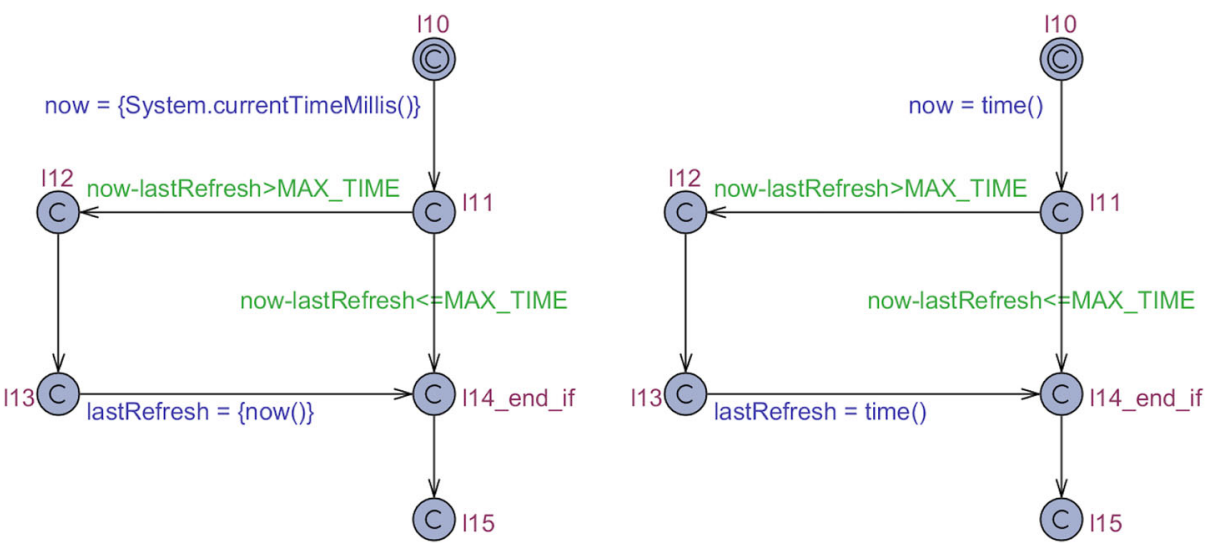

Fig. 4 The graph on the left depicts the timed automaton extracted from the source code of the read () method presented in Fig. 2. State names represent the source code line numbers. Expressions that are rewritten or replaced are contained between curly brackets. The graph on the right depicts an instance of this automaton after rewriting/replacing the marked expressions

The graph on the left hand side of Fig. 4 shows an example of this problem with the automaton extracted from the source code of the method read () displayed in Fig. 2. The calls to System.currentTimeMillis() and now() are surrounded by curly brackets to easily identify and later replace them. UPPAAL has no definition of these two Java methods and the three variables now, lastRefresh, and MAX_TIME. Therefore, we need to translate them into expressions that are processable by UPPAAL, whose details are discussed in the following subsections.

Time Variables In our approach, we model time variables defining them as UPPAAL variables using its integer theory. For time variables that are declared and initialized in the Java source code, our approach copies the Java initialization expression as initialization for the UPPAAL variables. Otherwise the UPPAAL variables are initialized with the value 0 . This however does not block our approach to correctly model the behavior of program variables that can be modified outside of the scope of the method. For instance, the variable lastRefresh is an instance variable that can be changed by other methods. We refer to these time variables as non-local time variables. They are handled by our approach separately, as we describe below.

RT Method Calls This category of method calls cannot be directly translated because UPPAAL does not come with a definition for those methods. For instance, it does not have a definition for the method System.currentTimeMillis(). In our manual analysis of Java 8 APIs, we identified which of Java's RT methods return a monotonic time value.

For these methods, we provide the UPPAAL defined function time that simulates the same behavior - every time it is called it returns a monotonically increasing natural number. Using this definition, our approach traverses the automaton and replaces all calls to such RT methods within curly brackets with a call to the time function.

Non-Local Time Variables and Unresolved Method Calls For the other method calls and non-local time variables contained in our automaton, our approach currently does not provide any formal model in UPPAAL. It addresses this limitation with dynamic analysis 
to monitor the program during its execution and to obtain representative values for these expressions. Then, our approach replaces these method calls and the initialization values of non-local time variables with the monitored values creating an automaton that can be model checked with UPPAAL.

Our approach performs the following steps: First, during the extraction of the automaton, our approach records the expressions that it could not translate into UPPAAL expressions. For each expression, our approach records the line number in the source file, the fully qualified names of the class and method containing that expression, and the expression itself. We implemented this method as an agent that is added to the Java Virtual Machine.

At the initialization phase of the Java Virtual Machine, the agent loads the list of recorded expressions and rewrites the bytecode of the classes that contain these expressions inserting logging statements. For the non-local time variables, a logging statement is inserted at the beginning of the method recording the value of each non-local time variable when the method is called. For each unresolved method call, it inserts the logging statement at the line where the method is called.

Each logging statement outputs the thread id, fully qualified names of the class and method, line number in the source code, the expression monitored, and the time value of the non-local time variable or method call to a log file. For each execution of a program such a $\log$ file is created.

Finally, for each thread and for each method execution, it creates an instance of the respective timed automaton replacing the non-local time variables and method calls with their recorded values.

The graph on the right hand side of Fig. 4 depicts an instance of a timed automaton created for the read () method in Fig. 2 with the correct syntax and semantic for the variables now and lastRefresh, and the System.currentTimeMillis () and now ( ) method calls. This automaton can be used with UPPAAL to formally verify the time properties of this method, such as termination.

\section{Evaluation}

In this section, we present the experiments we have performed to evaluate our approach to extract timed automata from the source code of Java methods. With the results we aim to answer the following five research questions:

- RQ1: What is the precision and recall of our approach to extract time information from source code?

- RQ2: What is the ratio of methods whose time behaviour depends on non-local time variables?

- RQ3: What is the number of method calls to time methods in external libraries?

- RQ4: Are the extracted models adequate to detect real errors in open source projects?

- RQ5: How much time and memory is required by our approach to extract the timed automata?

The first research question is used to evaluate the precision and recall of our approach to extract time information from source code. With respect to our time semantic defined for the Java 8 API, we expect our approach to extract this information with high precision and recall. The second and third research questions are used to evaluate the impact of two limitations of our approach, namely the usages of non-local time variables and external time 
libraries to implement time related functionality. The fourth research question investigates the capability of our approach to detect "real" errors in Java methods. Finally, the last research question studies how different metrics impact the time and memory needed by our approach to extract a timed automaton from a Java method. In the following, we first describe the dataset used in our experiments and then present the specific set-up for each research question and the results.

\subsection{Project Selection}

For our experiments, we used a set of 20 open source popular Java projects that have been also used in other time related studies (Liva et al. 2018).

ActiveMQ is a message broker and Activiti is a light-weight workflow and business process management platform. Airavata is a software suite to compose, manage, execute, and monitor large scale applications and workflows on computational resources. Alluxio enables any application to interact with any data from any storage system at memory speed. Atmosphere is a framework to develop client and server side components for building Asynchronous Web Applications and AWS-SDK-Java provides APIs to interact with many Amazon web services. Beam is a unified model for defining data-parallel processing pipelines and Camel is a framework to implement routing and mediation rules in Java- or Scala-based domain specific language. Elastic-Job is a project for running distributed scheduled jobs. Flume is a distributed service for collecting and aggregating log data. Hadoop is a map-reduce implementation and on top of its distributed file system, HBase builds a distributed database. Hazelcast is a clustering and highly scalable data distribution platform. Jetty is a web server provided by the Eclipse Foundation. Kafka provides a unified layer for handling real-time data feeds and, similarly, Lens provides a unified analytics interface from different data sources. Nanohttpd is a light-weight HTTP server designed for embedding in other applications and Neo4j is a graph database. Sling is a web framework that uses a Java Content Repository to store and manage content. Finally, Twitter4j is a Twitter API binding library for the Java language.

These projects use the Java time APIs for scheduling the communication between different components of a system, synchronize activities among different instances of a program, wait for an event to arrive, and handling communication failures over the network.

As can be seen by the descriptive statistics presented in Table 2, the size of the projects varies from 124 to 27,208 classes (column NC), whereas AWS-SDK-Java is the largest project. Only a small fraction of the methods implemented in each project contain a call to a Java 8 time method as indicated by the numbers in column NMT. In total, our semantics identified 11,772 time methods which yields a ratio of $1.24 \%$. The project with the largest number of methods that contain a call to a Java 8 time method is Hadoop with 2,686 methods. The project with the largest percentage of such methods is Flume with roughly $5 \%$ of the methods or 338 out of 6,705 methods. The set of 11,772 methods represent the basic input to our experiments.

\subsection{RQ1 - What is the Precision and Recall of Our Approach to Extract Time Information from Source Code?}

With this research question, we seek to evaluate the precision and recall of our approach in extracting time information from Java source code. The time information that can be extracted from source code are time constraints and time assignments that are then added to the timed automaton. 
Table 2 Open source Java projects used in our evaluation with number of Java files, number of classes (NC), number of methods (NM), and number of methods containing a call to a Java 8 SDK method implementing time (NMT)

\begin{tabular}{|c|c|c|c|c|c|}
\hline Project & Version & Files & $\mathrm{NC}$ & NM & NMT \\
\hline ActiveMQ & 5.16.0-SNAPSHOT & 4,434 & 5,071 & 43,520 & 1,480 \\
\hline Activiti & 7.0.0-SNAPSHOT & 2,002 & 2,103 & 15,373 & 66 \\
\hline Airavata & 0.17-SNAPSHOT & 1,621 & 9,320 & 70,862 & 154 \\
\hline Alluxio & 1.6.0-SNAPSHOT & 1,319 & 3,364 & 24,972 & 230 \\
\hline Atmosphere & 3.0.0-SNAPSHOT & 348 & 500 & 4,100 & 54 \\
\hline AWS-SDK-Java & 1.11.165-SNAPSHOT & 26,416 & 27,208 & 205,413 & 157 \\
\hline Beam & 2.2.0-SNAPSHOT & 1,696 & 3,844 & 21,401 & 85 \\
\hline Camel & 2.20.0-SNAPSHOT & 17,205 & 20,024 & 116,032 & 1,277 \\
\hline Elastic-Job & 2.1 .5 & 571 & 611 & 2,497 & 24 \\
\hline Flume & 1.8.0-SNAPSHOT & 642 & 995 & 6,705 & 338 \\
\hline Hadoop & 3.0.0-beta1-SNAPSHOT & 8,061 & 12,597 & 100,589 & 2,686 \\
\hline Hazelcast & 3.9-SNAPSHOT & 5,696 & 7,663 & 59,260 & 995 \\
\hline HBase & 3.0.0-SNAPSHOT & 3,638 & 9,535 & 128,914 & 1,500 \\
\hline Jetty & 9.4.7-SNAPSHOT & 2,567 & 3,781 & 25,548 & 980 \\
\hline Kafka & 0.11.1.0-SNAPSHOT & 1,315 & 1,896 & 14,006 & 326 \\
\hline Lens & 2.8.0-SNAPSHOT & 845 & 1,036 & 8,110 & 177 \\
\hline NanoHTTPD & 2.3.2-SNAPSHOT & 87 & 124 & 716 & 17 \\
\hline $\mathrm{Neo} 4 \mathrm{j}$ & 3.3.0-SNAPSHOT & 6,681 & 9,158 & 61,393 & 655 \\
\hline Sling & 9-SNAPSHOT & 5,336 & 5,964 & 37,415 & 530 \\
\hline Twitter4j & 4.0 .6 & 426 & 418 & 4,641 & 41 \\
\hline Total & & 90,906 & 125,212 & 951,467 & 11,772 \\
\hline
\end{tabular}

To evaluate the precision and recall of the generated timed automata, we follow the evaluation methodology presented by Le et al. (2015). First, the authors of the paper and an independent developer, who has several year of academic and professional experience in developing Java applications, create a reference set of timed automata. The reference set was created with a manual control- and data-flow analysis of 400 methods randomly selected from the 11,772 time methods. The manual data- and control-flow analysis has been performed independently by each researcher and the developer. The results and in particular the discrepancies were discussed in a follow-up meeting. For each discrepancy, the participants together analyzed the corresponding source code again until a consensus was reached. The resulting timed automata represent our ground truth models.

Precision and recall then were computed by comparing each timed automaton extracted by our approach with the respective ground truth model. Precision refers to the portion of time constraints and time assignments that were present in the extracted timed automaton that were also present in the ground truth model. Recall refers to the portion of time constraints and time assignments that were present in the ground truth model that were also present in the extracted timed automaton.

The result of this analysis shows a perfect precision and recall of $100 \%$. 


\subsection{RQ2 - What is the Ratio of Methods Whose Time Behaviour Depends on Non-Local Time Variables?}

Our approach extracts timed automata at method level but it does not consider time information stored in non-local time variables except for time constants. Based on our manual observations of the various methods in the 20 Java projects, we conjecture that developers tend to use time variables locally, except for time constants. The goal of this experiment is to verify our assumption by investigating to which extent developers use time variables locally, i.e., within methods, and to which extent non-local time variables are used and changed. An example of such a non-local time variable is given by the class attribute lastRefresh of class Cache in Fig. 2. In other words, we seek to investigate the ratio of pure methods w.r.t. time variables. Pure methods are methods that have the following two characteristics:

1. The result of a method only depends on the values of its parameters.

2. The execution of the method will not alter the value of any variable defined outside the scope of the method.

A pure method w.r.t. time variables has multiple benefits: they are secure, idempotent, easier to reason about, and easier to test, i.e., it is not necessary to set the system in a specific state since the result depends only on the input parameters.

For this experiment, we employ Ernst et al. (2007), an invariant detection tool. Daikon has been used in many previous research efforts, such as in (Beschastnikh et al. 2011, 2012, 2014, 2016; Abrahamson et al. 2014; Lemieux et al. 2015; Schiller and Ernst 2012; Baliga et al. 2011), to extract likely program invariants. It runs a program and observes the values that the program computes. During the observation, it applies some logic theories to infer which properties, i.e., invariants, are true over the observed execution. One of the many invariants extracted by Daikon concerns the values of variables. It detects if a variable never changes its value in a method during its execution. We can exploit this invariant to detect which non-local time variables are not modified in the execution of a method.

We randomly selected 400 methods from 11,242 time methods of the projects presented in Table 2. We excluded the 530 methods of Apache Sling because this project requires an external library that is also used by Daikon. Unfortunately, Apache Sling and Daikon depend on different versions of this library causing the tests of Sling to be not executable. Furthermore note, the list of 400 methods used for this research question is different from the list used in Section 5.2.

For each of the 400 methods, we ran the test suite of the respective project with Daikon and stored the invariants that it extracted. Next, we parsed the extracted invariants to check whether the method under study does alter the value of any non-local time variable. The results of this study show that only 19 out of $400(4.75 \%)$ methods alter the value of a non-local time variable while $95.25 \%$ (381/400) of the methods alter the value of a local time variable. This answers our research question RQ2: only $4.75 \%$ of the time methods alter the value of a non-local time variable during its execution. This low ratio confirms our hypothesis and we can conclude that developers mainly use local variables to implement time-related functionality. 


\subsection{RQ3 - What is the Number of Method Calls to Time Methods in External Libraries?}

Our approach relies on a time semantic that we defined for the Java 8 time APIs. However, developers might also use time APIs provided by other libraries, such as Joda-Time. ${ }^{3}$ Furthermore, they might use library methods which wrap the Java 8 time APIs. This might impact the precision, recall, and finally also the applicability of our approach since it does not support these libraries and the extracted models would miss this time information. With JSR-310, ${ }^{4}$ Java 8 improved the date and time APIs and we conjecture that developers rely only on them for handling time in their applications. With this research question, we want to verify our conjecture.

For each of the 20 projects in our data set, we performed the following steps: First, we collected the libraries used by the project using its build configuration. We erased the local repository used by Maven ${ }^{5}$ or Gradle ${ }^{6}$ and then executed the build process to download the libraries (i.e., jar files) used to build the project. They are stored by the build system into the local Maven or Gradle repository. Since the build system also copies the jar files created during the build into the local repository, we manually removed them to keep only the jar files of the libraries.

Next, we used the Fernflower ${ }^{7}$ Java decompiler to reconstruct the source code of each retrieved library of the project. Then, we applied our approach to the decompiled source code to detect public methods of the categories RT and ET. To collect the list of a library's time methods that encapsulate and export time functionalities, we have used the rules $R_{r t}$ and $R_{e t}$ of our approach, presented in Section 3.1.

With the two lists of Java 8 time methods and libraries' time methods, we next ran our prototype tool on the source code of the project to detect calls to the Java 8 time methods and calls to the libraries' time methods. To find out which concrete method of which class is called, we resolved the method bindings using the Eclipse $\mathrm{JDT}^{8}$ tools. The methods then were matched by their fully qualified name.

Table 3 presents descriptive statistics of the decompiled libraries. The number of libraries used by the 20 projects varies from 42 for Kafka to 2,611 for Camel, resulting in a total of 9,357 libraries to analyze. Decompiling the jar files of these libraries resulted in total in 1,308,284 Java source files implementing 1,870,583 classes and 18,329,808 methods. As expected, the source code decompiled from the libraries used by Camel contained the largest number of classes and methods, namely 612,571 classes and 6,540,417 methods.

Applying our prototype tool to analyze the 1,870,583 Java classes and 18,329,808 methods required more than 86 hours of computation. More than half of it (45.42 hours) was spent on analyzing the methods contained by libraries used by Camel. The analysis produced in total a list of 8,542 public time methods which is $0.047 \%$ of the methods. Camel alone contains half of the public time methods.

The last two columns of the Table 3 report the results of analyzing the calls to the Java 8 timed methods (JC) and libraries time methods (LC). The values in the column LC are

\footnotetext{
${ }^{3}$ http://www.joda.org/joda-time/index.html

${ }^{4}$ https://jcp.org/en/jsr/detail?id=310

${ }^{5}$ https://maven.apache.org/

${ }^{6}$ https://gradle.org/

${ }^{7}$ https://github.com/JetBrains/intellij-community/tree/master/plugins/java-decompiler/engine

${ }^{8}$ https://www.eclipse.org/jdt/
} 
Table 3 Statistics of the libraries used by the selected projects with number of libraries (\# Lib.), number of classes (\# Classes), number of methods (\# Methods), number of public time related method (\# PTM), hours of computation (Time), number of method calls to a Java time API (JC), and number of method calls to a library time method (LC)

\begin{tabular}{|c|c|c|c|c|c|c|c|}
\hline Project & \# Lib. & \# Classes & \# Methods & \# PTM & Time (h) & $\mathrm{JC}$ & $\mathrm{LC}$ \\
\hline ActiveMQ & 546 & 76,142 & 674,692 & 154 & 4.685 & 1,099 & 0 \\
\hline Activiti & 269 & 44,231 & 373,118 & 158 & 0.351 & 156 & 0 \\
\hline Airavata & 168 & 19,871 & 182,982 & 117 & 0.125 & 274 & 0 \\
\hline Alluxio & 298 & 45,254 & 426,670 & 179 & 0.307 & 338 & 0 \\
\hline Atmosphere & 128 & 8,525 & 68,434 & 53 & 0.064 & 56 & 0 \\
\hline AWS-SDK-Java & 161 & 18,803 & 173,239 & 126 & 0.172 & 234 & 0 \\
\hline Beam & 782 & 299,928 & $2,903,163$ & 29 & 20.161 & 123 & 0 \\
\hline Camel & 2,611 & 612,571 & $6,540,417$ & 4,474 & 45.420 & 585 & 0 \\
\hline Elastic-Job & 145 & 20,888 & 205,733 & 100 & 0.196 & 53 & 0 \\
\hline Flume & 522 & 113,117 & $1,086,062$ & 414 & 1.258 & 293 & 0 \\
\hline Hadoop & 431 & 66,282 & 571,628 & 319 & 0.624 & 2,798 & 0 \\
\hline Hazelcast & 223 & 36,149 & 291,106 & 100 & 0.287 & 1,055 & 0 \\
\hline Hbase & 429 & 80,956 & 765,446 & 188 & 5.316 & 1,642 & 0 \\
\hline Jetty & 562 & 60,443 & 547,066 & 42 & 3.799 & 527 & 0 \\
\hline Kafka & 37 & 7,401 & 68,511 & 34 & 0.139 & 417 & 0 \\
\hline Lens & 452 & 119,486 & $1,270,709$ & 593 & 0.972 & 152 & 0 \\
\hline NanoHTTPD & 118 & 7,188 & 54,121 & 41 & 0.054 & 7 & 0 \\
\hline Neo4j & 292 & 52,602 & 470,541 & 236 & 0.374 & 850 & 0 \\
\hline Sling & 1,091 & 165,453 & $1,469,600$ & 1,080 & 1.789 & 902 & 0 \\
\hline Twitter4j & 92 & 15,293 & 186,570 & 105 & 0.126 & 66 & 0 \\
\hline Total & 9,357 & $1,870,583$ & $18,329,808$ & 8,542 & 86.218 & 11,627 & 0 \\
\hline
\end{tabular}

all 0 , meaning that none of the 20 projects calls a method contained by a library that has been marked as time method by our semantics. This result clearly supports our conjecture and answers our research question RQ3: developers do not depend on methods in libraries to implement time-related functionality. They rely only on the Java time APIs.

\subsection{RQ4 - Are the Extracted Models Adequate to Detect Real Errors in Open Source Projects?}

In addition to the quantitative evaluation, we also performed an initial assessment of the effectiveness of our approach to detect time related bugs. For this, we manually investigated the Jira issue tracker of the Apache Software Foundation seeking for bugs that involve time.

We searched for the keyword timeout and applied filters to return bugs only for Java projects reported between January 1st, 2016 and January 1st, 2018. We manually filtered the results removing the bug reports that were not dealing with timing issues in the source code. The filtering was necessary because the majority of the bug reports returned by the query concern the adjustment of the timeout parameters for the integration test suite. Through this filtering, we obtained 8 reports, two from Flume, one from HBase, three from Kafka, and two from Lens. Note, 7 of the 8 bug reports come with an accepted patch that is attached to the report. At the moment of writing, one issue, namely FLUME-3044, was still open with 
Table 4 List of time-related bugs taken from the Apache issue tracker

\begin{tabular}{|c|c|c|}
\hline Issue ID & Version & Description \\
\hline FLUME-1401 & 1.3 .0 & Asynchbase sink should be configurable to support timeout. \\
\hline FLUME-3044 & 1.7 .0 & $\begin{array}{l}\text { There are several method call in } \\
\text { kafka sink with no timeout params, } \\
\text { in some cases, kafka sink will await } \\
\text { forever if no interruption. }\end{array}$ \\
\hline HBASE-17341 & 2.0 .0 & $\begin{array}{l}\text { In ReplicationSource.terminate(), a } \\
\text { Future is obtained from Replica- } \\
\text { tionEndpoint.stop(). Future.get() is } \\
\text { then called, but can potentially } \\
\text { hang there if something went wrong } \\
\text { in the endpoint } \operatorname{stop}() \text {. }\end{array}$ \\
\hline KAFKA-3540 & 0.10 .1 .0 & $\begin{array}{l}\text { Close the consumer, waiting indef- } \\
\text { initely for any needed cleanup. } \\
\text { That is not acceptable as it cre- } \\
\text { ates an artificial deadlock which } \\
\text { directly affects systems that rely } \\
\text { on Kafka A/I essentially rendering } \\
\text { them unavailable. }\end{array}$ \\
\hline KAFKA-4194 & 0.10 .1 .0 & $\begin{array}{l}\text { The loop below doesn't break when } \\
\text { the timeout is reached, right? It } \\
\text { will still block indefinitely until the } \\
\text { metadata has been refreshed. }\end{array}$ \\
\hline KAKFA-4306 & 0.10 .1 .0 & $\begin{array}{l}\text { If brokers are not available and we } \\
\text { try to shut down connect workers, } \\
\text { sink connectors will be stuck in } \\
\text { a loop retrying to commit offsets } \\
\text { forever. }\end{array}$ \\
\hline LENS-1032 & 2.5.0-beta & $\begin{array}{l}\text { We should provide option to kill the } \\
\text { query upon timeout for users who } \\
\text { are not interested in result beyond } \\
\text { timeout. }\end{array}$ \\
\hline LENS-1157 & 2.6 & $\begin{array}{l}\text { Execute with timeout fails to } \\
\text { read metadata when user requests } \\
\text { streamed results. }\end{array}$ \\
\hline
\end{tabular}

We present id, version affected, and a small summary of the issue description

a proposed patch attached to it. Table 4 presents the list of bugs and their short descriptions taken from their issue tracker summaries or github review comments.

Based on the bug descriptions, we divided the issues into two different types of errors:

- No Time-Bound: The method contains a loop that misses a time condition that sets a limit for its maximal execution time. The issues FLUME-3044, KAFKA-3540, KAFKA-4194, LENS-1032, and LENS-1157 belong to this category.

- Indefinite Wait: The method performs a call to an EW method, presented in Section 2.2, that potentially blocks the execution of a thread forever. The issues FLUME-1401, HBASE-17341, and KAFKA-4306 belong to this category.

We applied our approach to both, the original and patched versions of the code, and we ran our prototype tool to extract the timed automaton for each method that has been 
modified to fix the bug. Then, we used UPPAAL to first formally verify the existence of the reported bug and then its elimination in the patched version.

For the category No Time-Bound, we followed the methodology presented in Section 5.2 and created a set of ground truth transitions. For each method affected by this type of error, each researcher and the developer identified independently the transition that models the exit of the loop statement that causes the error. Then, we verified that the ground truth transition in the extracted automaton contains a time constraint that enforces a maximal execution time.

Concerning the category Indefinite Wait, we used UPPAAL to verify that each automaton can always terminate by executing the formula $A<>s_{i}$ where $s_{i}$ is the state that identifies an ending state in the CFG of the method. The formula checks whether the state $s_{i}$ can be eventually reached from the starting state. Our findings confirm the presence of all issues and furthermore the correctness of the proposed patches.

\subsection{RQ5 - How Much Time and Memory is Required by Our Approach to Extract the Timed Automata?}

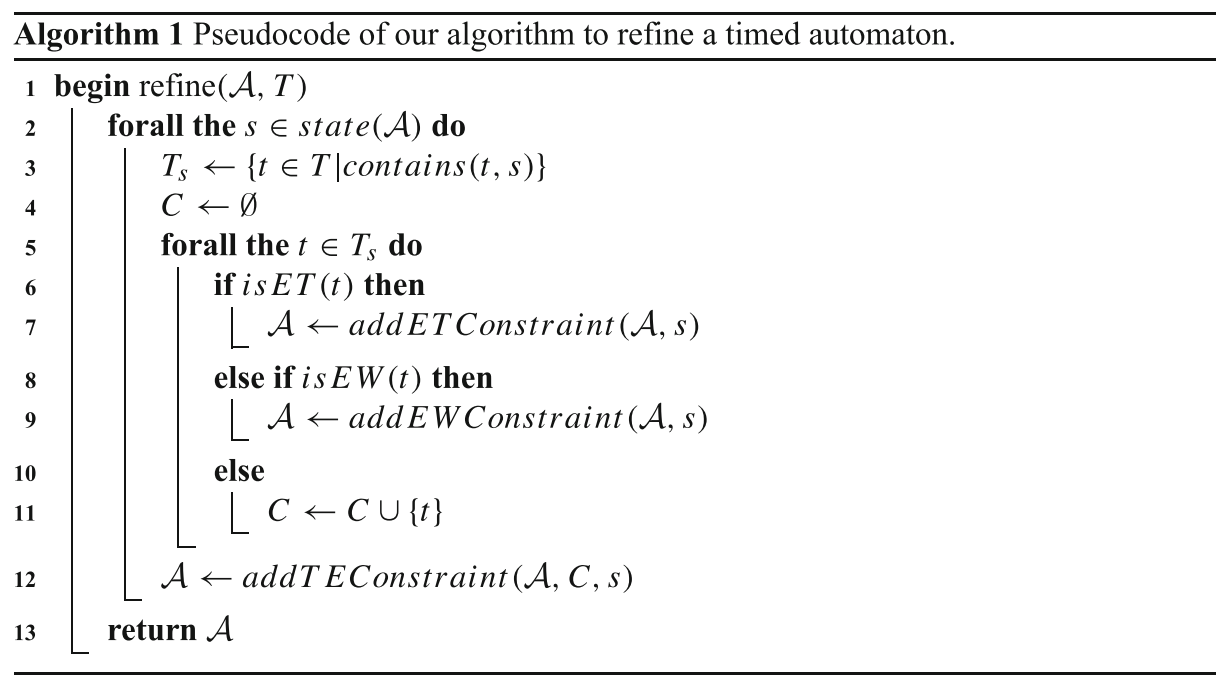

In this research question, we want to study how much time does our approach require to process the full source code of a project and how much time and memory it requires to extract the automaton for a single time method.

From a theoretical standpoint, the algorithm presented in Section 3 performs a greedy iteration over the statements of a method. The only exception is for statements that represent branching instructions, for which the algorithm iterates over the body of the branching instruction until a fix point is reached. Thus, the worst case scenario for this algorithm is a method in which every statement is a nested branching instruction. We denote $n$ as the number of statements of the method and $k$ as the length of the time information extracted, i.e., the number of elements stored in the environment $E$. We can conclude that, in the worst case, the time complexity to extract the time information for a method is $\mathcal{O}(n !)$. The algorithm constructs the output, i.e., list of the time information extracted, directly and no local memory is needed except for the fix point computation. Here, the algorithm needs to keep a copy 
of the environment computed in the previous iteration. Therefore, the space complexity to extract the time information from a method is $\mathcal{O}(k)$.

The algorithm to construct a timed automaton presented in Section 4 is divided into three different phases:

1. Construct an initial timed automaton.

2. Refine the automaton with time constraints.

3. Finalize the automaton with the runtime data.

In the first phase, the creation of the initial automaton is a one to one copy of the CFG representation into the UPPAAL automaton representation. Therefore, the creation of the automaton can be performed linearly in accordance with the size of the CFG. CFGs are a sparse type of graph since only maximum two transitions can depart from a node. We can conclude that the size of these graphs is bounded by $\mathcal{O}(n)$. This yields a time complexity of $\mathcal{O}(n)$ and a space complexity of $\mathcal{O}(1)$ for building the initial timed automaton.

We present the pseudo code of the second phase in Algorithm 1. The refine $(\mathcal{A}, T)$ function takes as input the automaton $\mathcal{A}$ created in the previous phase and the extracted time information $T$. The function attaches to every state of the automaton $\mathcal{A}$ its time constraint, if it exists. The retrieval of the time information for each state is performed at line 3 . This is achieved with a linear search on the list, yielding a time complexity of $\mathcal{O}(k)$. Then, for each time information of the state, the algorithm checks the type of time information and builds the correct time constraint for it, as presented in Section 4.2. Every statement between lines 6 and 11 is executed in $\mathcal{O}(1)$. In particular, if the time information $t$ is needed to generate the Time Expired constraint, we save it in the set $C$. Once all the time information for the Time Expired constraint are collected, they are processed by the function in line 12. The function addT EConstraint adds the Time Expired constrains for the state $s$ to the automaton $\mathcal{A}$. This is performed linearly in the size of $C$. Since each time information belongs to only one state, we can conclude that the worst case scenario is when all the time constraints are of type Time Expired. Thus, the time complexity for the function $\operatorname{refine}(\mathcal{A}, T)$ is the following:

$$
\begin{aligned}
T(n, k) & =\mathcal{O}(n) \times(\underbrace{\mathcal{O}(k)}_{\text {forall }}+\underbrace{\mathcal{O}(k)}_{\text {addT EConstraint }}) \\
& =\mathcal{O}(n k)
\end{aligned}
$$

For what it concerns the space complexity, the worst case scenario is when $C$ contains all the time information for every state, i.e., $|C|=\left|T_{s}\right|=\mathcal{O}(k)$.

The last phase consists of replacing missing information with the data collected through the runtime monitoring. This is performed with a greedy pass over the automaton transitions. Depending on the type of the expression in the transition, a different strategy is used, as presented in Section 4.3. Both Time Variables and RT Method calls are performed with a space and time complexity of $\mathcal{O}(1)$. The last category of expression, non-local time variables and unresolved method calls, requires to process the list of time information recorded with the runtime monitoring. This step requires a scan of the list to identify those values that belong to the current considered transition. The worst case scenario is when every single time information extracted needs to be monitored at runtime, yielding a time complexity of $\mathcal{O}(n k)$ and a space complexity of $\mathcal{O}(k)$. 
In summary, the time and space complexity to extract a timed automaton from a method with $n$ statements and $k$ time information are

$$
\begin{aligned}
T(n, k) & =\mathcal{O}\left(n^{n}\right)+\mathcal{O}(n)+\mathcal{O}(n k)+\mathcal{O}(n k) \\
& =\mathcal{O}\left(n^{n}\right)+\mathcal{O}(n k) \\
S(k) & =\mathcal{O}(k)+\mathcal{O}(1)+\mathcal{O}(k)+\mathcal{O}(k) \\
S(k) & \stackrel{\mathcal{O}}{=}(k)
\end{aligned}
$$

From the theoretical study of the space and time complexity, the time and memory required by our approach to produce a timed automaton heavily depends on the size and complexity of the source code implementing a method. Therefore, to empirically study the space and time complexity we consider the following three code metrics: (i) number of statements, (ii) McCabe cyclomatic complexity (CC), and (iii) number of variables used in the method. The number of statements indicates the size of a method. The cyclomatic complexity is a measure of the number of linearly independent paths through a program. We included it because the implementation of our approach iterates over branching instructions. Finally, we selected the number of variables as metric because our implementation keeps track of all variables in the environment and adds a special flag to the time related ones. Furthermore, when our implementation analyzes a branching statement, it creates a copy of the environment which is a time and memory consuming operation. We have computed these metrics based on the abstract syntax tree representation of the source code and they are available in our prototype tool.

Table 5 shows the minimum, median, and maximum values for the three code metrics measured for the 11,772 time methods per project. Statistics on the overall size of the projects are presented in Table 2. Over all projects, the time methods have a median size of 14 statements and a maximum size of 520 statements. The median cyclomatic complexity is 3 and the maximum complexity is 113 . The longest and most complex time method is found in the Jetty project. The time method using the highest number of variables is found in the Camel project. Overall, the number of variables ranges from 1 to 316 with a median value of 19 variables used per method.

We have performed our experiment to measure the time and memory consumption of our approach on a MacBook Pro with a $2.5 \mathrm{GHz}$ Intel i7 CPU, 16GB of main memory, running macOS 10.13.6.

The two columns on the left hand-side of Table 6 with the heading Full Project show the total time to process all methods of each project. Since our approach consists of two phases, we first computed the time required (using Eclipse JDT) to parse the source code into an Abstract Syntax Tree. Depending on the project size, our approach required from 3 seconds for parsing the source code of NanoHTTPD to more than 5.73 hours for parsing the source code of AWS-SKD-Java. Not surprisingly, the projects with the highest number of methods (see Table 2) also took the longest time to parse. For most of the projects, namely 16, the parsing took less than 13 minutes.

Regarding the second phase, the extraction of the timed automata, the results are presented in the column TA. For 16 projects, our approach extracted the timed automata in less than 17 minutes. Also for this phase NanoHTTPD took the least amount of time, namely 10 seconds, while AWS-SKD-Java took 1.9 hours.

Furthermore, since our approach computes the time constraints for only time methods, we also computed the time and memory used to produce the timed automata for these methods. We report the minimum, median, and maximum amount of time and memory per 
Table 5 Minimum, median, and maximum values of the source code metrics computed for the 11, 772 time methods of the 20 open source Java projects with number of statements (\# Statements), McCabe Cyclomatic Complexity (CC), and number of variables (\# Variables)

\begin{tabular}{|c|c|c|c|c|c|c|c|c|c|}
\hline \multirow[b]{2}{*}{ Project } & \multicolumn{3}{|c|}{ \# Statements } & \multicolumn{3}{|l|}{$\mathrm{CC}$} & \multicolumn{3}{|c|}{ \# Variables } \\
\hline & Min & Median & Max & Min & Median & Max & Min & Median & Max \\
\hline ActiveMQ & 1 & 19 & 182 & 1 & 3 & 61 & 1 & 18 & 136 \\
\hline Activiti & 1 & 17 & 282 & 1 & 4.5 & 86 & 2 & 13.5 & 72 \\
\hline Airavata & 1 & 19.5 & 390 & 1 & 6 & 46 & 4 & 19 & 73 \\
\hline Alluxio & 1 & 8 & 111 & 1 & 2 & 46 & 1 & 19 & 62 \\
\hline Atmosphere & 1 & 17 & 60 & 1 & 3 & 19 & 8 & 18.5 & 67 \\
\hline AWS-SDK-Java & 1 & 9 & 90 & 1 & 2 & 15 & 2 & 11 & 57 \\
\hline Beam & 1 & 13 & 61 & 1 & 4 & 21 & 2 & 22.5 & 57 \\
\hline Camel & 1 & 11 & 113 & 1 & 2 & 43 & 1 & 11 & 316 \\
\hline Elastic-Job & 3 & 6 & 26 & 1 & 3 & 6 & 5 & 10 & 22 \\
\hline Flume & 1 & 19 & 108 & 1 & 3 & 58 & 5 & 20 & 109 \\
\hline Hadoop & 1 & 16 & 437 & 1 & 3 & 81 & 2 & 23 & 131 \\
\hline Hazelcast & 1 & 10 & 58 & 1 & 2 & 18 & 1 & 16 & 294 \\
\hline Hbase & 1 & 18 & 192 & 1 & 4 & 81 & 2 & 26 & 220 \\
\hline Jetty & 1 & 19 & 520 & 1 & 2 & 113 & 4 & 18 & 103 \\
\hline Kafka & 1 & 12 & 110 & 1 & 3 & 42 & 1 & 26.5 & 312 \\
\hline Lens & 1 & 16 & 105 & 1 & 3 & 27 & 5 & 18 & 96 \\
\hline NanoHTTPD & 1 & 8 & 57 & 1 & 1 & 18 & 5 & 8 & 41 \\
\hline $\mathrm{Neo} 4 \mathrm{j}$ & 1 & 9 & 77 & 1 & 2 & 24 & 2 & 14 & 102 \\
\hline Sling & 1 & 14 & 162 & 1 & 3 & 47 & 2 & 17.5 & 74 \\
\hline Twitter4j & 1 & 9.5 & 109 & 1 & 2 & 42 & 5 & 16 & 65 \\
\hline Overall & 1 & 14 & 520 & 1 & 3 & 113 & 1 & 19 & 316 \\
\hline
\end{tabular}

project in the columns on the right hand side of Table 6. In addition, we also show the distributions of the time and memory consumption with violin plots in Fig. 5.

Note, these results comprise both, the parsing and the extraction of the timed automaton for a given time method. Looking at the table and the violin plots, we can see that the time required to process a method varies from $98 \mathrm{~ms}$ to $2857 \mathrm{~ms}$ with a median value of $192 \mathrm{~ms}$ using between 3 and $89 \mathrm{MB}$ of memory with a median value of $9.5 \mathrm{MB}$. With respect to the median time, the time methods in the Hadoop project took the longest to process, namely $233 \mathrm{~ms}$, while the time methods in the Elastic-Job project took the least amount of time, namely $115 \mathrm{~ms}$. The maximum amount of time to process a time method was in the Hadoop project, namely $2857 \mathrm{~ms}$. The violin plot for Time in Fig. 5 shows that for $93.25 \%$ of the time methods, our tool required less than $381 \mathrm{~ms}$.

With respect to the median memory consumption, the processing of the time methods in Airavata consumed the highest amount of memory, namely $12 \mathrm{MB}$. Instead, the time methods in Hadoop required the minimum but also the maximum amount of memory with $3 \mathrm{MB}$ and $89 \mathrm{MB}$, respectively. Looking at the memory violin plot in Fig. 5, 95.37\% of time methods required less than $18 \mathrm{MB}$ of memory to be processed by our prototype tool.

In addition to the distribution of time and memory, we also investigated the correlation between the three code metrics and time and memory consumption. Since none of our five 
Table 6 Time required to parse (Parsing) and extracting the timed automata (TA), along with the minimum, median, and maximum amount of Time and Memory required by our prototype tool to generate a timed automaton

\begin{tabular}{|c|c|c|c|c|c|c|c|c|}
\hline \multirow[b]{2}{*}{ Project } & \multicolumn{2}{|c|}{ Full project (s) } & \multicolumn{3}{|c|}{ Time (ms) } & \multicolumn{3}{|c|}{ Memory (KB) } \\
\hline & Parsing & TA & Min & Median & Max & Min & Median & Max \\
\hline ActiveMQ & 158 & 381 & 133 & 229 & 816 & 6,715 & 9,066 & 54,540 \\
\hline Activiti & 434 & 962 & 106 & 139 & 319 & 6,518 & 7,085 & 54,170 \\
\hline Airavata & 645 & 687 & 112 & 211 & 447 & 5,422 & 12,090 & 31,720 \\
\hline Alluxio & 154 & 291 & 122 & 171 & 401 & 6,035 & 8,523 & 40,070 \\
\hline Atmosphere & 23 & 81 & 136 & 182 & 392 & 7,225 & 9,056 & 22,910 \\
\hline AWS-SDK-Java & 20,635 & 6,846 & 107 & 151 & 352 & 5,353 & 7,125 & 28,540 \\
\hline Beam & 165 & 207 & 116 & 166 & 345 & 6,967 & 7,760 & 18,410 \\
\hline Camel & 4,071 & 1,918 & 105 & 145 & 471 & 3,791 & 10,160 & 31,350 \\
\hline Elastic-Job & 16 & 37 & 107 & 115 & 147 & 7,223 & 7,657 & 10,160 \\
\hline Flume & 36 & 79 & 125 & 211 & 376 & 7,306 & 9,300 & 18,920 \\
\hline Hadoop & 1,379 & 1,657 & 113 & 233 & 2,857 & 3,041 & 10,220 & 89,060 \\
\hline Hazelcast & 524 & 1,068 & 102 & 185 & 442 & 5,231 & 9,740 & 26,030 \\
\hline Hbase & 1,394 & 924 & 114 & 212 & 808 & 5,122 & 9,359 & 51,850 \\
\hline Jetty & 228 & 423 & 111 & 185 & 466 & 6,869 & 10,110 & 32,160 \\
\hline Kafka & 90 & 328 & 107 & 176 & 336 & 6,339 & 10,020 & 34,510 \\
\hline Lens & 56 & 193 & 106 & 165 & 503 & 6,707 & 9,876 & 31,280 \\
\hline NanoHTTPD & 3 & 10 & 112 & 123 & 247 & 7,567 & 7,763 & 25,110 \\
\hline $\mathrm{Neo} 4 \mathrm{j}$ & 589 & 981 & 98 & 144 & 453 & 5,393 & 7,264 & 34,010 \\
\hline Sling & 751 & 762 & 109 & 157 & 1,410 & 5,975 & 8,749 & 28,370 \\
\hline Twitter4j & 24 & 91 & 121 & 170 & 302 & 7,389 & 9,233 & 15,190 \\
\hline Overall & 31,374 & 17,926 & 98 & 192 & 2,857 & 3,041 & 9,549 & 89,060 \\
\hline
\end{tabular}

metrics have a normal distribution, we used the Spearman's rank method to compute the correlation. A Spearman $\rho$ value of +1 and -1 indicates high positive or high negative correlation, whereas 0 indicates that the variables under analysis do not correlate at all. Values greater than +0.3 and lower than -0.3 indicate a moderate correlation; values greater than +0.5 and lower than -0.5 are considered to be large/strong correlations (Hopkins 2014).

The strongest correlation is shown between the cyclomatic complexity and the time, with a $\rho$ of 0.54 . This means an increase in the complexity of a method most likely leads to an increase in the time needed to extract the timed automaton for that method. Instead, the cyclomatic complexity shows only a weak correlation with the memory consumption, namely 0.26 . The number of variables moderately correlates with both, time and memory, with a Spearman's $\rho$ of 0.48 . Finally, also the number of statements moderately correlates with the time and the memory consumption with a $\rho$ of 0.38 and 0.36 , respectively. All the correlation coefficients are significant with a p-value lower than 0.01 .

We can conclude that our approach can extract the timed automata for the majority of projects in less than half an hour. Furthermore, if a developer narrows down the scope to a specific method, our approach performs the extraction in less than $381 \mathrm{~ms}$ using roughly $18 \mathrm{MB}$ of memory in $95 \%$ of the cases. The cyclomatic complexity of the method impacts 

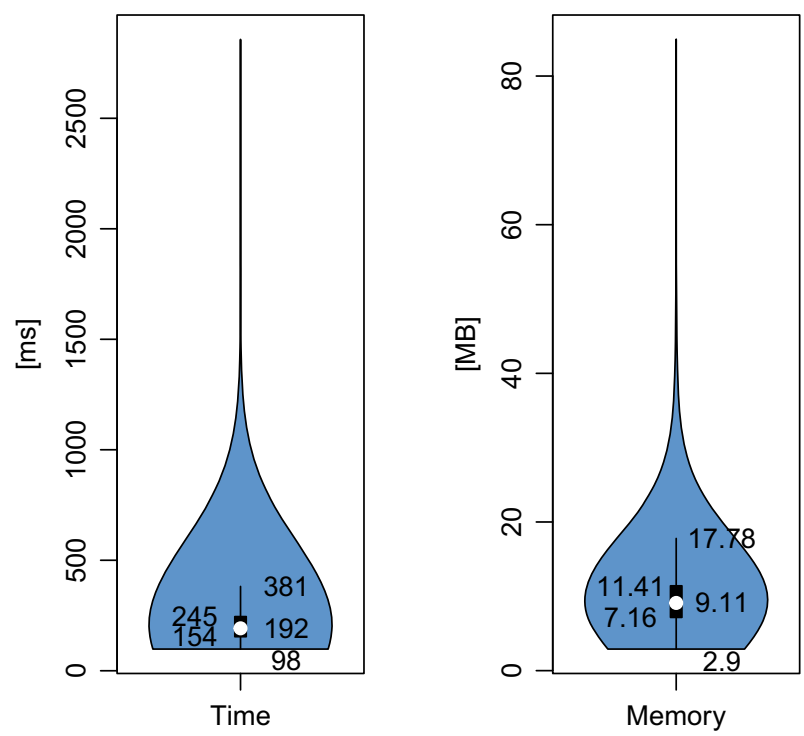

Fig. 5 Violin plots with the distribution and the quartiles of time and memory consumption

the processing time the most, followed by the number of variables. The latter is also the factor that most likely impacts the memory consumption.

\section{Discussion}

This section discusses the results and their implications on research and practice. Furthermore, we discuss the limitations of our current approach and the potential threats to validity of our empirical findings.

\subsection{Summary of Results}

In Section 3, we proposed a time semantics for Java that we used to develop an approach to automatically extract timed automata from the source code of a Java method. We presented this approach in Section 4 and its evaluation in Section 5.

A key property of an automated approach is that it should be sound and complete. We investigated this property with our research questions RQ1, RQ2, and RQ3. The results of research question RQ1 show a precision and recall for our time semantics of $100 \%$.

The results of research question RQ2 show that, for $95 \%$ of the time methods, our approach could extract timed automata that model the entire time behavior of those methods. Only for 5\% of the methods a dynamic analysis step was needed to add the missing time information that could not be extracted or modeled through our static analysis approach. But, also this step is automated by our approach and does not need any human intervention. The shortcoming, however, is that the created instances of the timed automata for these methods do not cover all possible values for the time variables used in a method but only the values created through running the test cases. Consequently, while only affecting $5 \%$ of the methods, our translation from Java source code to UPPAAL is not complete. 


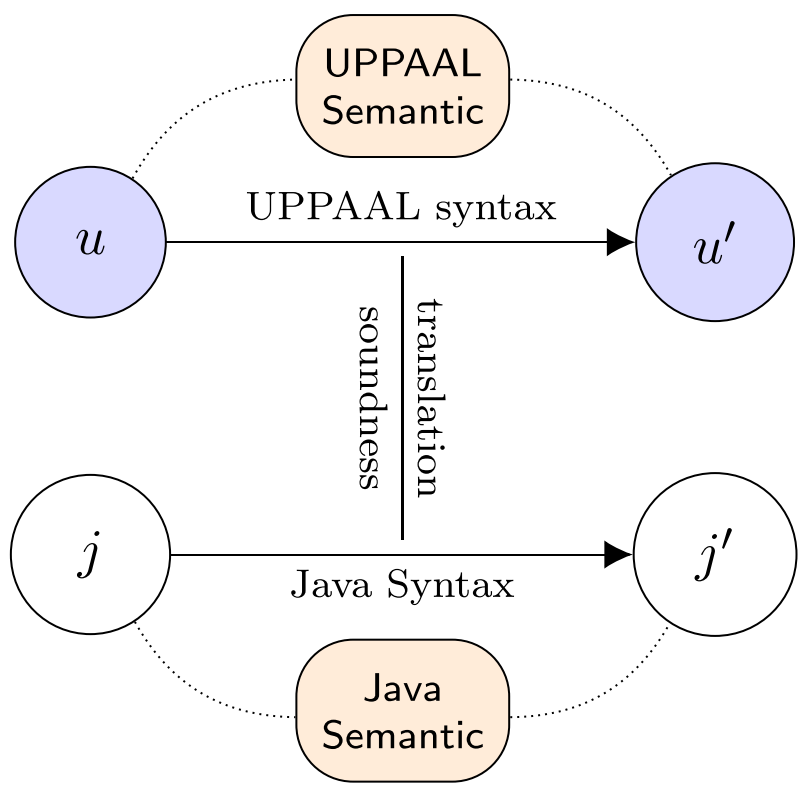

Fig. 6 Sketch of the proof of soundness of our translation from Java source code to UPPAAL

Concerning the soundness, RQ1 shows a perfect precision and recall that supports a sound translation. The diagram in Fig. 6 presents a sketch of the proof of soundness of our translation. In fact, the formal proof assures that any time-transition $\left(j\right.$ to $\left.j^{\prime}\right)$ of a given Java program has the equivalent time semantics applied by UPPAAL in processing the transition from the respective state $u$ to state $u^{\prime}$. In other words, we assure that our UPPAAL correctly simulates any valid transition of its Java counterpart w.r.t. time. In principle, the proof of soundness is a structural induction proof based on the Java statement that involve time, whose further details are beyond the scope of this paper.

The results of research question RQ3 clearly show that developers exclusively use the Java 8 time APIs to implement time-related functionality. They do not use methods provided in third party libraries that are not covered by our time semantics.

Summing up the results of RQ1, RQ2, and RQ3, we view our approach to be sound and complete, at least with respect to our time semantics for the version 8 (and later versions) of the Java time APIs.

The results of research question RQ4 show that our extracted timed automata can be effectively used to detect time related errors and to confirm the correctness of the proposed patches. Finally, from the results of research question RQ5 we can conclude that the runtime complexity of our approach scales with the size and complexity of the project, requiring 30 minutes to process most of the projects.

\subsection{Implications of Results}

Concerning the implications on the research in this area, our approach improves existing approaches that model time as a sequence of events represented in a tree-like structure, such as presented by Walkinshaw and Bogdanov (2008). These approaches fail to model time aspects of a program, such as timing delays. Furthermore, our approach does not require 
developers to manually craft a timed automaton to formally verify the time behavior of a method, such as presented by Jayaraman et al. (2015). This prevents the introduction of human errors.

The formal definition of a semantics for the time domain can be used by other formal verification tools, such as Java Path Finder (Havelund and Pressburger 2000), to verify a richer spectrum of properties, such as concurrency defects like deadlocks, and unhandled exceptions like Null PointerExceptions and AssertionErrors. In addition, our semantics can be used to investigate techniques that can help developers to find time related problems in early phases of the development. We recently presented an approach (Liva et al. 2018) that uses our time semantics encoded in the Z3 SMT solver (Deharbe et al. 2011; Barrett et al. 2010) to identify values for time variables that cause a program to fail.

Our results also have several implications on practitioners. Software developers and testers can use our approach to automatically extract timed automata to verify the implementation of time-related functionality in Java programs. Developers can specify time properties that must hold during the development of the system and through that discover errors in the implementation of time-related functionality of modified Java methods. Currently, our implementation does not exploit multi-processors capabilities and the total time to process a project can be further reduced parallelizing the analysis. However, on our laptop machine the process of a project required half an hour with low memory footprint. Therefore, developers could integrate our approach into the pipeline of their continuous integration systems to analyze each new version of a program committed to the source code repository.

Furthermore, the extracted timed automata can be used during code reviews to help the reviewers analyze the correctness of the implementation. For instance, reviewers can run queries with the UPPAAL model checker to verify that the implementation satisfies the specification.

\subsection{Limitations}

In our manual analysis of the Java 8 APIs presented in Section 2.2, we discovered four different categories of time methods. Currently, we do not provide any abstraction that can model methods of the category ST where a method call has a time behavior if and only if a timeout is set by a preceding method call. For instance, the connection method of the URLConnection class by default has no upper time limit for establishing the connection. Instead, a timeout can be set by calling the setConnectTimeout method before calling the connection method. We plan to address this limitation in our future work.

While we consider EW methods of the Java 8 time APIs, we do not gather the project's EW methods. Although they are time related methods, they do not reference any time variable and currently, we do not provide any rule that infers them from the statical analysis of the source code. Future work will be devoted to infer this kind of time related methods through abstract interpretation or dynamic analysis to discover patterns that identify methods which can possibly block the execution of a program forever. Furthermore, we model EW method calls with a self-loop without considering its synchronization with external events. This limitation could be overcome by considering a network of timed automata. Due to the inheritance and polymorphism offered by Java, multiple automata can send a message to the EW state. Considering all possibilities is not a valid option since it may not be representative of the implementation. We plan to address this limitation complementing our current approach with a dynamic analysis that monitors the program and identifies the 
sender and receiver instructions. Then, we could simulate this behavior in the network of timed automata forcing the corresponding states to communicate via channels.

Currently, our approach requires a developer to manually specify which property must hold in the extracted automata. Once she/he has defined them, our approach can automatically verify those. We plan to address this limitation in our future work by automatically inferring the properties to verify from the test suite of a project.

\subsection{Threats to Validity}

In the following, we discuss threats to the internal and external validity of our evaluation, and how we addressed them in our experiments.

Internal Validity One threat to internal validity concerns the reliability of our prototype implementation. We mitigated this threat by testing the prototype tool manually and with unit tests. For each analysis in RQ1 and RQ2, we randomly selected 400 methods that were used to evaluate our approach. The size of our sample set is larger than the minimum number (372) required to obtain results at a $95 \%$ confidence level with a 5\% margin of error. Moreover, we showed the applicability of our approach on 8 bugs taken from Apache open source projects (RQ4). Our approach confirmed the existence of the bugs and also validated the correctness of the proposed patches.

Another threat concerns the possibility that our approach might miss to model the behavior stemming from time methods that are provided or wrapped by third party libraries. With the research question RQ3 we evaluated the impact of this threat. We set up an empirical study with 20 open source Java projects where we investigated the number of public methods of the projects' libraries that implement time-related functionality and are called by methods in the 20 Java projects. The results of our study show that only $0.047 \%$ of the methods in the libraries export time functionalities. Moreover, none of them is used in the source code of the 20 projects. We therefore can conclude that developers do not depend on methods in libraries to implement time-related functionality but they rely only on the Java time APIs that our approach extracts with $100 \%$ precision and recall.

The manual analysis to create the ground truth for answering RQ1 and RQ4 poses another threat to internal validity. We mitigated it by asking also an independent developer to perform the manual analysis. Each discrepancy then has been discussed with all participants until a consensus was reached.

Java does not permit to directly allocate or free memory. This could introduce a threat to the validity of the results of RQ5. We mitigated this threat by calling the Garbage Collector (GC) before applying our approach to remove the extra memory allocated by the JVM. Since the call to the GC is async, we poll the method call until it is eventually invoked by the JVM. We have used the APIs offered by the ManagementFactory class to monitor when the release of the spurious memory is indeed executed.

External Validity Threats to external validity concern the generalization of the results to other software projects. We mitigated this threat by choosing 20 open source Java projects that differ in size and domains to improve the generalization of our results. We also implemented our approach in a prototype tool that is publicly available online and can be applied to other Java projects to extend our studies. Furthermore, our formal time semantics can be adapted to other programming languages, such as $\mathrm{C \#}$, that use a semantics of time simil ar to Java. 
Furthermore, in RQ2 we relied on Daikon to extract invariants that identify pure methods w.r.t. time. We configured Daikon to monitor the execution of the tests provided with the projects. Its results heavily depend on the characteristics of the test suite and, therefore, they might not be representative for every possible input value of the tested methods. However, tests cover how a specific piece of code should be executed and the results extracted by Daikon can be considered representative use cases for the method under analysis. Further studies are required to additionally verify this generalization, complementing Daikon with static approaches and with automatic unit test generators, e.g., Randoop (Pacheco and Ernst 2005), to execute the method with additional input.

\section{Related Work}

One of the main contributions of this paper is our time semantics that we have used to determine which Java statements are time related. In the domain of semantics for the Java programming language, Bogdanas and Roşu (2015) present a formal semantics for Java version 1.4 based on their $\mathbb{K}$-Framework (Roşu and Serbảnutả 2010). They formalize the language syntax and how the Java Virtual Machine (JVM) interprets the bytecode. Similarly, the Real-Time for Java Expert Group (Bollella and Gosling 2000) provide a specification, called RTSJ, that enforces a specific semantics for the Java Virtual Machine and introduces a new set of APIs. They specify how the JVM should interpret specific classes to enable the creation, verification, analysis, execution, and management of Java threads for real time programs. However, their focus is on the scheduling of threads for reaching a real-time execution and not on verifying the time-related functionality implemented in a program.

The second contribution of this paper is an approach to extract timed automata from source code. There are existing works that translate source code to timed automata. Cicirelli et al. (2013) present a library for UPPAAL that is able to reproduce the semantics of major Java concurrent and synchronization mechanisms.

Yang et al. (2016) present a tool that translates Simulink Stateflow models into UPPAAL timed automata for verification. With the verification power of UPPAAL, their approach manages to find design defects that were missed by the Simulink Design Verifier. Timed automata are also used as basic design specification to verify properties in a program. The approach presented by Jayaraman et al. (2015) takes as input a network of timed automata provided by developers. The network is used as base knowledge of the specific real-time system. They monitor the execution of a program verifying that its behavior conforms to the network of timed automata provided. In an analogous way, Hakimipour et al. (2010) propose a technique to automatically generate a program from timed automata. They use a timed automaton to produce an RTSJ program that is executable on single- and multi-processor platforms. Georgiou et al. (2013) present a technique to translate timed input/output automata into distributed executable Java programs. These approaches require developers to manually construct such time models which could introduce errors. Furthermore, these approaches do not guarantee that the models are an exact representation of the implementation. Our approach, on the contrary, automatically extracts models from the implementation that are directly amenable to the verification.

In the domain of verifying properties of source code, NASA developed Java Path Finder (Havelund and Pressburger 2000), a framework for verification and debugging of Java programs. The tool is used to verify properties of Java programs with a focus on race conditions. 
It converts the bytecode of a program into the Promela model checking language. Similarly, Henzinger et al. (2002), present a framework for verifying properties of C programs for the mutex API. Hatcliff and Dwyer (2001) automatically extracts a state machine from Java source code amenable to verification. In all the previous approaches, the models represent the control and data flow of a program without taking into account the specifics of the domain of time. The work of Walkinshaw and Bogdanov (2008) describes an extension of an existing state machine inference technique in which it accounts for temporal properties of the subjected system. However, it does not consider time explicitly but only represents it as a sequence of events as they happened in the execution of a program. On the contrary, our approach considers the specific time semantics of the programming language that models more time properties, such as timing delays.

\section{Conclusions}

In this paper, we presented an approach to automatically extract timed automata from the source code of a Java program. We first introduced a definition of the semantics for time in the Java programming language. The time semantics is then used by our approach to extract the timed automata.

Our approach first identifies Java statements related to time, second, it infers time constraints, and lastly, it uses this information to create the timed automata.

We have implemented our approach in a prototype tool that was used to answer five research questions. First, we empirically studied the soundness and completeness of our time semantics manually analyzing 400 Java methods randomly selected from 20 open source Java projects. The results of the manual evaluation show that our time semantics can detect time statements and time variables with $100 \%$ precision and recall (see Section 5.2).

In the second research question we studied the ratio of methods for which our approach could extract a timed automaton that entirely models the time behavior. The results show that this worked for $95 \%$ of the methods. For the remaining 5\% of methods our approach performed an additional dynamic analysis step to create an instance of the timed automaton for each test case that then can be used for verification (see Section 5.3).

With the third research question we found that our time semantics is adequate to describe all the time APIs that are used by developers and it does not need to consider time methods defined in external libraries (see Section 5.4).

Furthermore, with the fourth research question, we presented 8 examples of real bug reports of four Apache projects. We used our approach to verify the existence of the bugs as well as the correctness of their patches (see Section 5.5). With the last research questions, we showed that the median time for extracting a timed automaton is 0.192 seconds and the total time to process most of the projects is 30 minutes (see Section 5.6).

Our results benefit researchers and practitioners. In addition to automating the extraction of timed automata, our time semantics can be used by other formal verification tools to verify a richer spectrum of properties. Furthermore, developers can benefit from our research, for instance by integrating our approach into their continuous integration system to verify that the time specification is correctly implemented.

Future work will be dedicated to improve our approach to provide a model also for methods of the category ST. Moreover, we plan to extend our prototype tool to support annotations that allow developers to manually mark the time category of their methods. Currently, developers have to encode their requirements in UPPAAL. Future work will be devoted to remove this manual step and infer them automatically from the test suite of their 
projects. Furthermore, we plan to extend our approach to other programming languages, such as C\#. And finally, we will extend our studies to cover a larger set of open source projects and also industrial projects. Concerning industrial projects, we also plan to evaluate the usefulness of our approach with software developers and testers, and apply our approach to real-time systems.

Acknowledgments This research is funded by the Austrian Research Promotion Agency FFG within the FFG Bridge 1 program, grant no. 850757.

Funding Information Open access funding provided by University of Klagenfurt.

Open Access This article is distributed under the terms of the Creative Commons Attribution 4.0 International License (http://creativecommons.org/licenses/by/4.0/), which permits unrestricted use, distribution, and reproduction in any medium, provided you give appropriate credit to the original author(s) and the source, provide a link to the Creative Commons license, and indicate if changes were made.

\section{References}

Abadi M, Cardelli L (2012) A theory of objects. Springer Science \& Business Media

Abrahamson J, Beschastnikh I, Brun Y, Ernst MD (2014) Shedding light on distributed system executions. In: Companion proceedings of the 36th international conference on software engineering. ACM, pp 598-599

Alur R (1999) Timed automata. In: International conference on computer aided verification (CAV). Springer, pp 8-22

Alur R, Dill DL (1994) A theory of timed automata. Theor Comput Sci 126(2):183-235

Baier C, Katoen JP, Larsen KG (2008) Principles of model checking. MIT Press

Baliga A, Ganapathy V, Iftode L (2011) Detecting kernel-level rootkits using data structure invariants. IEEE Trans Depend Sec Comput 8(5):670-684

Barrett C, Stump A, Tinelli C et al (2010) The SMT-LIB standard: version 2.0. In: Proceedings of the 8th international workshop on satisfiability modulo theories (Edinburgh, England), vol 13, p 14

Behrmann G, David A, Larsen KG (2004) A tutorial on UPPAAL. In: Formal methods for the design of real-time systems. Springer, pp 200-236

Bengtsson J, Griffioen WD, Kristoffersen KJ, Larsen KG, Larsson F, Pettersson P, Yi W (1996) Verification of an audio protocol with bus collision using UPPAAL. 1102. Springer, pp 244-256. http://www.docs. uu.se/docs/rtmv/papers/bgkllpw:cav96.ps.gz

Beschastnikh I, Brun Y, Schneider S, Sloan M, Ernst MD (2011) Leveraging existing instrumentation to automatically infer invariant-constrained models. In: Proceedings of the 19th ACM SIGSOFT symposium and the 13th European conference on foundations of software engineering. ACM, pp 267-277

Beschastnikh I, Brun Y, Ernst MD, Krishnamurthy A, Anderson TE (2012) Mining temporal invariants from partially ordered logs. ACM SIGOPS Oper Syst Rev 45(3):39-46

Beschastnikh I, Brun Y, Ernst MD, Krishnamurthy A (2014) Inferring models of concurrent systems from logs of their behavior with CSight. In: Proceedings of the 36th international conference on software engineering. ACM, pp 468-479

Beschastnikh I, Wang P, Brun Y, Ernst MD (2016) Debugging distributed systems. Queue 14(2):50

Bogdanas D, Roşu G (2015) K-Java: a complete semantics of Java. In: Proceedings of the ACM SIGPLANSIGACT symposium on principles of programming languages (POPL). ACM, pp 445-456

Bollella G, Gosling J (2000) The real-time specification for Java. Computer 33(6):47-54

Börger E, Fruja NG, Gervasi V, Stärk RF (2005) A high-level modular definition of the semantics of C\#. Theor Comput Sci 336(2-3):235-284

Cardelli L, Gordon AD (1998) Mobile ambients. In: International conference on foundations of software science and computation structure. Springer, pp 140-155

Cicirelli F, Furfaro A, Nigro L, Pupo F (2013) Modelling Java concurrency: an approach and a UPPAAL library. In: Federated conference on computer science and information systems (FedCSIS). IEEE, pp 1373-1380 
Deharbe D, Fontaine P, Paleo BW (2011) Quantifier inference rules for SMT proofs. In: First international workshop on proof eXchange for theorem proving (PxTP)

Dijkstra EW (1972) The humble programmer. Commun ACM 15(10):859-866

Ernst MD, Perkins JH, Guo PJ, McCamant S, Pacheco C, Tschantz MS, Xiao C (2007) The Daikon system for dynamic detection of likely invariants. Sci Comput Program 69(1-3):35-45

Farzan A, Chen F, Meseguer J, Ros่u G (2004) Formal analysis of Java programs in JavaFAN. In: International conference on computer aided verification. Springer, pp 501-505

Filaretti D, Maffeis S (2014) An executable formal semantics of PHP. In: European conference on objectoriented programming. Springer, pp 567-592

Flatt M, Krishnamurthi S, Felleisen M (1998) Classes and mixins. In: Proceedings of the 25th ACM SIGPLAN-SIGACT symposium on principles of programming languages. ACM, pp 171-183

Georgiou C, Musial PM, Ploutarchou C (2013) Tempo-toolkit: tempo to Java translation module. In: International symposium on network computing and applications (NCA). IEEE, pp 235-242

Guth D (2013) A formal semantics of Python 3:3

Hakimipour N, Strooper P, Wellings A (2010) TART: timed-automata to real-time Java tool. In: International conference on software engineering and formal methods (SEFM). IEEE, pp 299-309

Hatcliff J, Dwyer M (2001) Using the Bandera tool set to model-check properties of concurrent Java software. In: International conference on concurrency theory (CONCUR). Springer, pp 39-58

Hathhorn C, Ellison C, Rosiu G. (2015) Defining the undefinedness of C. In: ACM SIGPLAN notices, vol 50. ACM, pp 336-345

Havelund K, Pressburger T (2000) Model checking Java programs using Java pathfinder. Int J Softw Tools Technol Transfer (STTT) 2(4):366-381

Hennessy M (1988) Algebraic theory of processes. MIT press

Henzinger TA, Necula GC, Jhala R, Sutre G, Majumdar R, Weimer W (2002) Temporal-safety proofs for systems code. In: International conference on computer aided verification (CAV). Springer, pp 526-538

Hopkins WG (2014) A new view of statistics. Internet Society for Sport Science

Hune T, Larsen KG, Pettersson P (2001) Guided synthesis of control programs using UPPAAL. Nord J Comput 8(1):43-64

Jayaraman S, Hari D, Jayaraman B (2015) Consistency of Java run-time behavior with design-time specifications. In: International conference on contemporary computing (IC3). IEEE, pp 548-554

Khan MT, Serpanos D, Shrobe H (2018) ARMET: behavior-based secure and resilient industrial control systems. Proc IEEE 106(1):129-143

Laporte CY, Nabil B, Mikel D (2012) Measuring the cost of software quality of a large software project at bombardier transportation: a case study. Softw Qual Manage 14(3):14-31

Larsen KG, Pettersson P, Yi W (1995) Diagnostic model-checking for real-time systems. In: Proc. of workshop on verification and control of hybrid systems III, 1066. Springer, pp 575-586

Larsen KG, Pettersson P, Yi W (1997) UPPAAL in a nutshell. Int J Softw Tools Technol Transfer (STTT) 1(1-2):134-152

Le TDB, Le XBD, Lo D, Beschastnikh I (2015) Synergizing specification miners through model fissions and fusions (t). In: 2015 30th IEEE/ACM International conference on automated software engineering (ASE). IEEE, pp 115-125

Lemieux C, Park D, Beschastnikh I (2015) General LTL specification mining (t). In: 2015 30th IEEE/ACM International conference on automated software engineering (ASE). IEEE, pp 81-92

Lindahl M, Pettersson P, Yi W (1998) Formal design and analysis of a gear-box controller. In: Proc.of the 4th workshop on tools and algorithms for the construction and analysis of systems, no. 1384 in lecture notes in computer science. Springer, pp 281-297

Liva G, Khan MT, Pinzger M (2017) Extracting timed automata from Java methods. In: Proceedings of the IEEE 17th international working conference on source code analysis and manipulation (SCAM), pp 91-100

Liva G, Khan MT, Spegni F, Spalazzi L, Bollin A, Pinzger M (2018) Modeling time in Java programs for automatic error detection. In: Proceedings of the IEEE/ACM conference on formal methods in software engineering (FormaliSE 2018). IEEE Press

Lo D, Mariani L, Pezzè M (2009) Automatic steering of behavioral model inference. In: Proceedings of the the 7 th joint meeting of the European software engineering conference and the ACM SIGSOFT symposium on the foundations of software engineering (ESEC/FSE). ACM, pp 345-354

McCarthy J (1964) A formal description of a subset of ALGOL. Tech. rep., Stanford University Department of Computer Science

McCarthy J (1993) Towards a mathematical science of computation. In: Program verification. Springer, pp 35-56 
Milner R (1999) Communication and mobile systems: the $\pi$-calculus. Cambridge Uni

Pacheco C, Ernst MD (2005) Eclat: automatic generation and classification of test inputs. In: 19th European conference on ECOOP 2005 — object-oriented programming. Glasgow, pp 504-527

Park D, Ştefănescu A, Roşu G (2015) KJS: a complete formal semantics of Javascript. In: Proceedings of the 36th ACM SIGPLAN conference on programming language design and implementation (PLDI'15). ACM, pp 346-356

Plotkin GD (1977) LCF considered as a programming language. Theor Comput Sci 5(3):223-255

Plotkin GD (1981) A structural approach to operational semantics. Computer Science Department Aarhus University Denmark

Roşu G., Serbảnutȧ TF (2010) An overview of the K semantic framework. J Logic Algebraic Program 79(6):397-434

Sangiorgi D, Walker D (2003) The pi-calculus: a theory of mobile processes. Cambridge University Press

Schiller TW, Ernst MD (2012) Reducing the barriers to writing verified specifications. ACM SIGPLAN Not 47(10):95-112

Stärk RF, Schmid J, Börger E (2012) Java and the Java virtual machine: definition, verification, validation. Springer Science \& Business Media

Steel T (1969) A formalization of semantics for programming language description. In: Proc. IFIP WG formal language description languages for computer programming, pp 25-36

Tristan JB (2009) Formal verification of translation validators. Ph.D. thesis Université Paris 7 Diderot

Walkinshaw N, Bogdanov K (2008) Inferring finite-state models with temporal constraints. In: Proceedings of the IEEE/ACM international conference on automated software engineering (ASE). IEEE Computer Society, pp 248-257

Wright AK, Felleisen M (1994) A syntactic approach to type soundness. Inf Comput 115(1):38-94

Yang Y, Jiang Y, Gu M, Sun J (2016) Verifying Simulink Stateflow model: timed automata approach. In: Proceedings of the IEEE/ACM international conference on automated software engineering (ASE). ACM, pp $852-857$

Yovine S (1997) Kronos: a verification tool for real-time systems. Int J Softw Tools Technol Transfer 1(12): $123-133$

Publisher's note Springer Nature remains neutral with regard to jurisdictional claims in published maps and institutional affiliations.

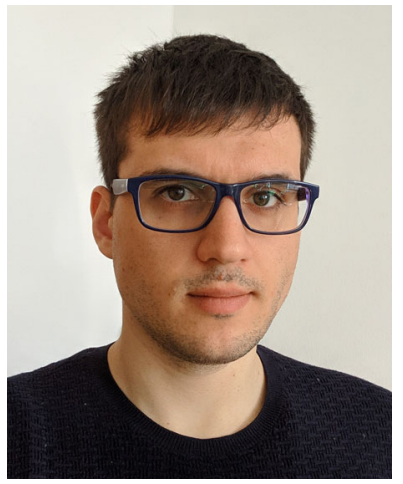

Giovanni Liva is working toward the doctoral degree at the AlpenAdria Universität, Austria. He received his Computer Science B.Sc. in 2013 at Universitá degli Studi di Udine. In 2015 he received his M.Sc. degree cum laude in the joint program between Universitá degli Studi di Udine and Alpen-Adria-Universität Klagenfurt. His research interests include program analysis, abstract interpretation, model checking, and software evolution. 

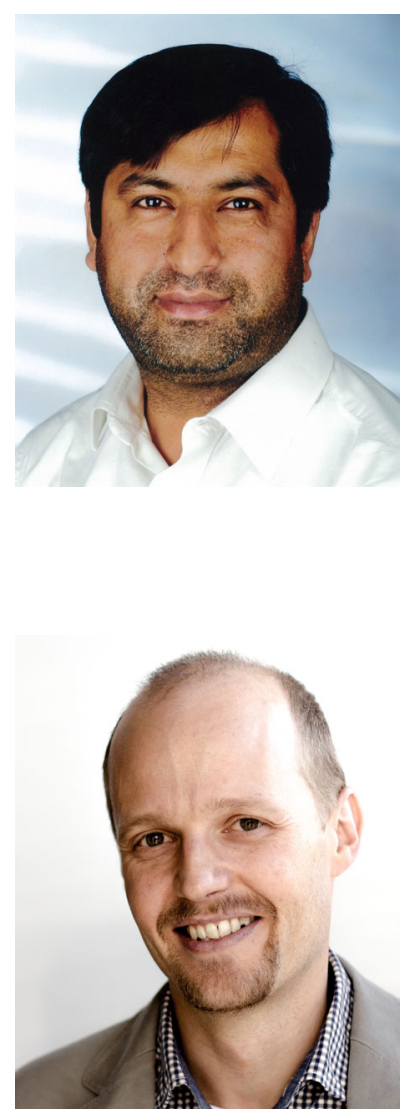

Muhammad Taimoor Khan is Lecturer in Secure Systems at Surrey Center for Cyber Security, University of Surrey, UK. His current research focus is on developing secure by design cyber physical (robotic) systems. Since last decade, he has been applying formal methods to assure reliability and security of various software systems, e.g., industrial control systems, computer mathematics-based systems, to name a few. He has been working as a scientist at various premier international research institutes, including INRIA, France and MIT CSAIL, USA; he is jointly working with these institutes now. Dr. Khan has won various research and academic awards including best paper award(s).

Martin Pinzger is a full professor at the University of Klagenfurt, Austria where he is heading the Software Engineering Research Group. His research interests are in software evolution, mining software repositories, program analysis, software visualization, and automating software engineering tasks. He is a member of ACM and a senior member of IEEE. 